\section{Antenatal exposure to antidepressant drugs and the risk of neurodevelopmental and psychiatric disorders: a systematic review}

\author{
Exposição intrauterina a antidepressivos e risco \\ de transtornos de neurodesenvolvimento e \\ psiquiátricos: uma revisão sistemática
}

\section{Exposición prenatal a medicamentos antidepresivos y riesgo de trastornos psiquiátricos y en el desarrollo neurológico: una revisión sistemática}

Jessica Salvador Areias de Araujo 1,2 Isabella Fernandes Delgado 2

Francisco José Roma Paumgartten 1

\begin{abstract}
This study investigated whether antenatal exposure to antidepressants (ADs) increases the risks of autism spectrum disorders (ASD), attention deficit/hyperactivity disorders (ADHD), schizophrenia and other mental illnesses, and cognitive and developmental deficits in infants or preschool children. PubMed, EMBASE, BIREME/BVS databases were searched to identify studies examining associations of $A D$ s in pregnancy with neurodevelopmental and psychiatric disorders. Twenty studies addressed ASD and/or ADHD risks while 30 focused on developmental and cognitive deficits in infants or preschool children. Most studies detected no association of antenatal AD with ASD after adjustment of risk ratios for maternal depression or psychiatric disorders. Some studies showed that maternal depression, regardless of whether it is treated or untreated, increased ASD risks. Seven out of 8 studies found no increase in $A D H D$ risk associated with antenatal exposure to selective serotonin reuptake inhibitors, the most commonly used AD. No consistent evidence was found linking $A D$ in pregnancy to neurocognitive developmental deficits in infants or preschool children. A residual confounding by indication (depression severity) remained in almost all studies. This systematic review found no consistent evidence suggesting that $A D$ s in pregnancy increase risks of $A S D, A D H D$, and neurocognitive development deficits. Some studies, however, found evidence that maternal depression increases ASD risks.
\end{abstract}

Antidepressant Drugs; Depression; Autism Spectrum Disorders; Attention Deficit Disorder with Hyperactivity; Pregnancy

\section{Correspondence}

F. J. R. Paumgartten

Laboratório de Toxicologia Ambiental, Escola Nacional de Saúde Pública Sergio Arouca, Fundação Oswaldo Cruz. Av. Brasil 4036, sala 101-104, Rio de Janeiro, RJ 21040-361, Brasil.

paum@ensp.fiocruz.br

${ }_{1}^{1}$ Escola Nacional de Saúde Pública Sergio Arouca, Fundação Oswaldo Cruz, Rio de Janeiro, Brasil.

2 Instituto Nacional de Controle de Qualidade em Saúde, Fundação Oswaldo Cruz, Rio de Janeiro, Brasil. 


\section{Introduction}

Depression is a common but serious disorder characterized by a constellation of symptoms, namely, insomnia or hypersomnia, anhedonia, feelings of worthlessness, deep sadness and excessive guilt, extreme fatigue, loss of energy, diminished ability to concentrate and to make decisions, loss or increase of appetite and/or weight, psychomotor retardation or agitation, and suicidal thoughts and attempts 1 .

In 2015, the World Health Organization (WHO) estimated that 322 million people, or 4.4\% of world's population, suffered from depression 2. By 2030 depression will become the leading cause of global burden of disease as measured by the disability-adjusted life years (DALY) 2. Depression is nearly 1.7 -fold more common in females than in males and vulnerability to it increases in pregnancy and puerperium when many women experience their first depressive episode, or have a recurrent manifestation of a previously diagnosed disorder 3. It affects up to $10 \%$, or even more, of pregnant women and mothers who have recently given birth 4 . Although depression is treatable, only approximately $12 \%$ of depressed pregnant women receive adequate treatment 5 .

There is little consensus about the management of depression in pregnancy and reluctance to prescribe antidepressants (ADs) to pregnant women arises mostly from unresolved concerns regarding their risks to the unborn child 6 . Accumulated evidence from observational investigations and meta-analyses indicates that maternal use of selective serotonin reuptake inhibitors (SSRI) in the first trimester of gestation entails a slightly higher risk of congenital malformations, particularly of heart defects ${ }^{7}$. Some studies revealed associations of use of non-SSRIs with adverse pregnancy outcomes other than congenital anomalies, such as preterm delivery, low birth weight and perinatal complications 8,9. A recent systematic review, however, found no consistent differences between AD-treated and untreated patients in the risk level for poor pregnancy outcomes, such as small-for-gestationalage babies, spontaneous abortions and preterm births 10 .

Concerns about harmful effects of maternal depression and its pharmacological treatment on the unborn child go beyond the endpoints evaluated at term pregnancy and neonatal period. It is thought, for instance, that antenatal exposure to ADs might increase the risk of neurodevelopmental disorders such as autism spectrum (ASD) and attention-deficit/hyperactivity (ADHD) disorders, and/or cognitive deficits 11 . This notion is plausible because the fetal brain undergoes a process of rapid cell proliferation and migration, synaptogenesis and circuitry formation. Considerable experimental and clinical evidence apparently links dysfunctions of serotoninergic transmission to disruption of neural network shaping and subsequent appearance of neurodevelopmental disorders 12,13. It is of note that the most commonly prescribed antidepressants inhibit, either selectively or non-selectively, serotonin uptake from the synaptic cleft. Serotonin acts as a nervous tissue growth factor and, by doing this, it modulates neural plasticity and network formation in the developing brain 13. Serotonin-dependent shaping of neural circuitry provides an insight into a possible mode of action by which antenatal exposure to serotonin reuptake inhibitors might increase the risk of neurodevelopmental disorders. Along this line, Rotem-Kohavi et al. 14 recently reported findings of a possible neural correlate of epidemiologic associations between antenatal exposure to SSRIs and neurodevelopmental disorders. Using a resting-state functional magnetic resonance imaging, the authors noted that, compared with healthy control infants and infants whose depressed mothers did not receive antidepressants, newborns prenatally exposed to SSRIs exhibited a hyperconnectivity in auditory resting-state networks.

Several cohort and case-control studies addressed a potential association between antidepressant drug use by pregnant women and neurodevelopmental disorders such as ASD and ADHD in their children 15. These studies arrived at conflicting conclusions about whether or not antenatal exposure to antidepressants is associated with ASD or ADHD. There is far less information about risks of neurodevelopmental disorders other than ASD and ADHD, such as mental illnesses of later onset and long-term cognitive deficits.

Eight reviews of observational studies showed a positive association between exposure to ADs (SSRIs) in utero and ASD 16,17,18,19,20,21,22,23, whereas two studies found no evidence of association or inconsistent findings 24,25 . Whether these associations were causal, however, remained unclear. Three reviews evaluated whether prenatal $\mathrm{AD}$ exposure was associated with ADHD and found no evidence 
of a causal link 25,26,27. Only one recent review examined whether AD use in pregnancy was associated with neurodevelopmental outcomes other than ASD and ADHD 28.

Autistic disorders are as a rule diagnosed around 3-4 years of age, and last throughout the individuals' life 29. They are characterized by symptoms such as deficits of social interaction and communication, limited and repetitive patterns of behavior, poor eye contact, lack of response to his/her name and/or indifference to caregivers, difficulty to express his/her emotions or feelings, apparent indifference to the feelings of others, inability to start a conversation or keep one going, and others 29 . Some children with ASD have learning disabilities whereas others show normal to high intelligence, yet having great difficulty to communicate or apply what they learned. Both genetic and environmental factors seem to contribute to ASD susceptibility and severity 29 . According to current estimates, ASD affects approximately $1 \%$ of people, being more frequently diagnosed in boys than in girls. There is no effective therapy for ASD, nor are there effective means to prevent it 29. Identification of environmental risk factors for ASD is, therefore, a public health goal of utmost importance.

ADHD is characterized by difficulty in paying attention and or hyperactivity/impulsivity 30 . It is about 2 -fold more frequent in boys than in girls, generally diagnosed in children under the age of 12 years, and lasts throughout the persons' life. Most patients have combined attention deficit and hyperactivity symptoms, and their enhanced distractibility and impulsivity impairs school performance and social skills 30,31. Although ADHD pathophysiology remains unclear, its symptoms show a good response to treatment with dopaminergic drugs. ADHD is one of the most prevalent childhood psychiatric disorders, affecting 5-7\% children, when diagnosed by DSM-IV criteria, and 1-2\% when diagnosed by WHO's (ICD-10) criteria 30,31. Overdiagnosis and overtreatment of ADHD, however, is a matter of debate.

The objective of this review was to answer a question of relevance for both public health and clinical practice: Is there epidemiologic evidence suggesting that prenatal exposure to ADs increases risks of ASD, ADHD, other psychiatric disorders of later onset and neurodevelopmental deficits in the exposed offspring? A well-founded response to this question would enable physicians and health authorities to make informed decisions about the use of antidepressant drugs to treat maternal depression in pregnancy. A corollary aim of the systematic review was to disclose research gaps requiring further epidemiology studies.

This study adds to existing reviews due to its broader research question, encompassing ASD, ADHD, psychiatric disorders of later onset and other neurodevelopmental outcomes, and because it reviewed a greater number of observational studies and critically appraised their methodological limitations.

\section{Methods}

This review followed the recommendations of the Preferred Reported Items for Systematic Reviews and Meta-Analyses (PRISMA) guidelines 32, and was registered with PROSPERO 2018 (CRD42018080950).

\section{Search strategy and study selection}

A systematic search on PubMed, EMBASE, BIREME/BVS (Virtual Health Library - Brazil) electronic databases identified epidemiologic studies addressing the association of ADs in pregnancy with neurodevelopmental disorders in the offspring. The search covered the period between the inception of the database and December 2nd, 2018 (PubMed and BIREME/BVS), or January 1st, 2019 (EMBASE). The BIREME/BVS database includes articles published in periodicals indexed in the Latin American and Caribbean Literature on Health Sciences (LILACS). We also conducted a manual screening of reference lists of articles, reviews, and other documents, to identify additional studies potentially eligible for reviewing. There was no restriction regarding the language of the article. The search strategy combined Medical Subject Heading (MeSH; https://www.ncbi.nlm.nih.gov/mesh) terms for the pharmacological classes of antidepressants (with their variations) with pregnancy (or gestation) and with MeSH 
terms for neurodevelopmental disorders and cognitive skills (and variations). To design the search strings, Boolean connectors "AND" and "OR" were used for combining the search terms. Term combinations and the full search strategy are shown in Figure 1 and Supplementary Material - Appendix 1 (http://cadernos.ensp.fiocruz.br/site/public_site/arquivo/suppl-app1-e00026619_3711.pdf).

\section{- Inclusion criteria}

Studies were eligible for inclusion if they investigated, in children born to mothers with any exposure to AD in pregnancy, outcomes such as diagnoses of ASD, ADHD, mental disorders (schizophrenic, affective or anxiety disorders), or emotional, internalizing and externalizing behaviors, speech and language, intelligence, neuromotor development, or any other form of cognitive functioning, assessed at least 3-4 weeks after birth, using scales or any other method. Internalizing behaviors (or disorders) are children's negative behaviors characterized primarily by processes focusing inward (on the self) such as anxiety, fearfulness, social withdrawal, somatization and depression. Contrasting with internalizing behaviors, externalizing behaviors are directed outwards (the external world) such as hostility, antisocial behavior, and aggression.

\section{- Exclusion criteria}

Articles were not eligible for reviewing if they met any of the exclusion criteria, namely, in vivo studies conducted in animals, in vitro/ex-vivo investigations, ecologic and non-analytic epidemiology studies, cross-sectional studies, case-reports, case-series, letters, editorials, reviews, meta-analyses, notes, comments, clinical guidelines, opinion papers, full-paper not available, and articles not available in English, Portuguese, Spanish, German or French.

As shown in Figure 2, selection of retrieved studies (after excluding duplicates) for reviewing was a two-step screening process: at first, publications were screened by titles and abstracts and, if deemed potentially eligible, at a subsequent phase full articles were retrieved and read. Two reviewers independently screened retrieved studies for eligibility and, if they did not reach a consensus after extensive discussion, a third reviewer was asked to resolve it.

\section{Figure 1}

Design of systematic review search strings (MeSH terms) with Boolean connectors "AND" and "OR".

MATERNAL EXPOSURE

Serotonin Uptake Inhibitors OR 5-HT Uptake Inhibitors OR

5-Hydroxyrryptamine Uptake Inhibitors OR Selective Serotonin Reuptake Inhibitors OR Serotonin Reuptake Inhibitors

Serotonin and Noradrenaline Reuptake Inhibitors OR SNRIs OR

SSRIS and NRIS OR

Serotonin and Norepinephrine Reuptake Inhibitors OR

Serotonin and Norepinephrine Uptake Inhibitors

Antidepressant Drugs, Tricyclic OR

Antidepressants, Tricyclic OR

Tricyclic Antidepressive Agents
OFFSPRING OUTCOMES

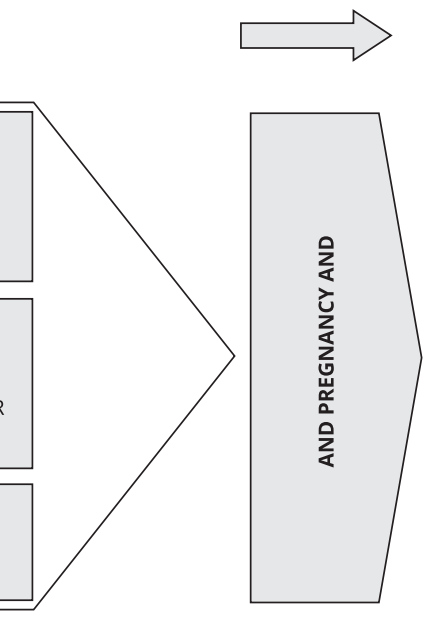

Anxiety disorder + OR Bipolar disorder OR Depressive disorder OR Cyclothymic disorder OR Motor disorder OR Neurocognitive disorder + OR Neurodevelopmental disorder OR Attention deficit and disruptive behavior disorders OR Attention deficit disorder with hyperactivity OR Conduct disorder OR Child behavior disorder OR Child behavior disorder, pervasive OR Asperger syndrome OR Autism spectrum disorder OR Autistic disorder OR Communication disorder OR Developmental disabilities OR Intellectual disability OR Learning disorders OR Motor skills disorders OR Schizophrenia, childhood OR Schizophrenia spectrum OR Affective disorders, psychotic OR Paranoid disorders OR Schizoprenia 


\section{Figure 2}

PRISMA flowchart of selection of studies for inclusion in the review.

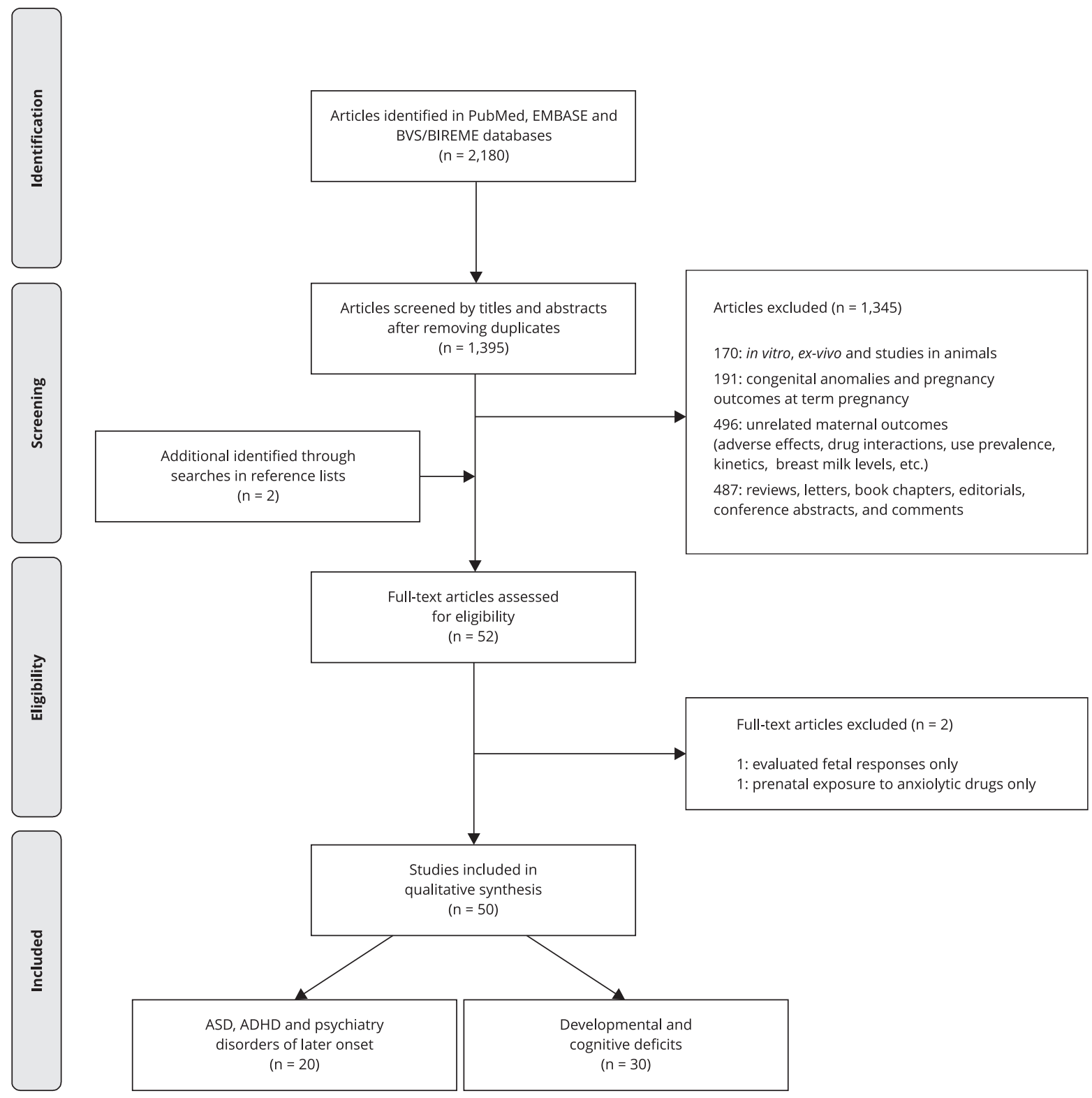

ADHD: attention deficit/hyperactivity disorder; ADs: antidepressants; ASD: autism spectrum disorders.

\section{Data extraction}

Based on the STROBE statement checklist of items that should be included in reports from observational studies 33 , two reviewers extracted a predetermined set of data from each study selected for review. Extracted data included first author's name, publication year, geographic location, study period, sample size, drug exposure (type of antidepressant and pharmacological class), period of exposure during pregnancy, strengths and limitations of the study, findings, outcome with risk estimates 
and $95 \%$ confidence intervals (95\%CI), adjusted confounders, and study conclusions. A third reviewer examined the compiled data and study summaries, and differences between reviewers were resolved with discussion.

\section{Assessment of the methodological quality}

The assessment of study quality was based on the Newcastle-Ottawa Scale (NOS) for observational (case-control and cohort) epidemiology studies 34 . NOS consists of eight items grouped into three domains for selection, comparability and outcome and two reviewers independently assessed each of the studies assigning a score (maximum score $=9$ ) to the study quality. Any discrepancy between reviewers was resolved by a third reviewer.

\section{Data synthesis}

For each outcome evaluated, we present the characteristics and findings of all included studies. A qualitative (narrative) synthesis of the evidence for ASD, ADHD and other neurodevelopmental outcomes responded the research question. It took into account not only the results of the reviewed studies but also their methodological limitations and residual confounding. We considered undertaking a quantitative synthesis for ASD and ADHD. Nonetheless, we did not perform a meta-analysis because of the heterogeneity in design and characteristics of the included studies and the fact that several studies (national-based cohorts) used the same national data source (registry) with overlapping of data collection times.

\section{Results}

As shown in the PRISMA flow diagram of study selection process (Figure 2), of the 50 studies included in the review, 20 addressed risks of ASD and ADHD while 30 investigated risks of developmental and cognitive impairments in infants, toddlers and preschool children. One Finnish study investigated the impact of gestational exposure to SSRIs on offspring psychiatric disorders including not only ASD and ADHD, but also anxiety and depression in early adolescents 35 .

\section{Autism spectrum disorders}

Five out of 16 studies evaluating whether prenatal ADs increased risks of ASD found no increase in risk 35,36,37,38,39. Three studies detected a weak association of AD in pregnancy with ASD that were no longer significant when risk estimates were adjusted for confounding factors such as maternal depression or history of psychiatric disorders 40,41,42. Hagberg et al. 43 found a significant association of ASD with antenatal AD, when the unexposed offspring (no AD, no history of depression) was compared with a group exposed to both $\mathrm{AD}$ and maternal depression in pregnancy. No increase in risk of ASD was evident, however, when unexposed offspring was compared with offspring of pregnant women who received $\mathrm{AD}$ for disorders other than depression 43 . Six cohort and case-control (nested in population-based cohorts) investigations, on the other hand, found a modest association of antenatal AD with ASD 44,45,46, ASD in boys 47, and ASD without intellectual disability 48,49. One of the reviewed studies evaluated whether antenatal exposure to SSRI was associated with higher scores for autistic traits in children 50. El Marroun et al. 50 found a modest association between maternal (self-reported) use of SSRIs in pregnancy and higher scores for autistic traits in infants assessed by child behavior checklists and a social responsiveness scale. Since depressed women are likely to overestimate problems with their children, and a residual confounding by indication of SSRIs (severity of depression) remained, associations of SSRI use in pregnancy with higher scores for autistic traits in infants may be non-causal. 


\section{Attention deficit/hyperactivity disorders}

Four out of 8 studies that examined possible associations between AD in pregnancy and ADHD found no association between exposure and outcome 35,37,42,51. Three studies revealed no association of ADHD with maternal use of SSRIs, yet they found that this disorder was - weakly to moderately - associated with prenatal exposure to non-SSRIs, tricyclic ADs (TCA) and atypical antidepressants.

Figueroa 52 found that maternal use of SSRIs and ADs other than bupropion in pregnancy did not increase the risk of ADHD in children aged 5 years or younger. Antenatal bupropion, on the other hand, was moderately associated with ADHD especially when exposure occurred in the 2 nd trimester of gestation. Bupropion inhibits both norepinephrine (NE) and dopamine (DA) reuptakes and, additionally, releases these neurotransmitters into the synaptic cleft. It also acts as a nicotinic receptor antagonist 53 . This atypical antidepressant is used in combination with another AD, when patients are resistant to SSRI, and as an adjunct therapy for smoking cessation, weight loss and ADHD 54,55,56. Boukhris et al. 57 found that TCA in the $2 \mathrm{nd} / 3$ rd trimesters of pregnancy was weakly associated with ADHD, even after adjustment for maternal history of depression. A territory population-based study by Man et al. 58 found that non-SSRI use in pregnancy was associated with a modest increase in the risk of ADHD in the offspring, yet a subsequent sibling analysis showed no association.

Finally, a case-control study by Clements et al. 41 found a modest association of ADHD with prenatal exposure to SSRIs \& non-SSRIs, especially during the 1st trimester of gestation. This association was significant even after adjustment of risk estimates for maternal history of depression.

\section{Quality assessment}

The quality of cohort and case-control studies selected for review ranged from fair to very good (NOS scores 6 to 9), and 16 and 8 of them investigated risks of ASD and/or ADHD, respectively, in children born to AD-treated pregnant women (Tables 1 and 2). The reasons for lower assessments (fair quality) were lower scores in Selection (2) and Comparability (1), and Outcome (1) and Comparability (1) domains for one case-control 44 and one cohort 50 study, respectively (Supplementary Material - Appendix 2: http://cadernos.ensp.fiocruz.br/site/public_site/arquivo/suppl-app2-e00026619_ 2540.pdf).

\section{Mental disorders of later onset}

Malm et al. 35 found that prenatal exposure to SSRIs modestly increased risks of depression in the offspring (SSRI exposed vs. non-exposed, after adjustment for maternal psychiatric disorder; $\mathrm{HR}=$ 1.78; 95\%CI: 1.12-2.82). In this Finnish national register-based cohort study, cumulative incidence of diagnoses of depression was determined up to adolescence (age of 14) 35. Notwithstanding the attempt to adjust risk estimates for records of maternal history of psychiatric illnesses, the severity of maternal depressive disorder remained as a residual confounding. It is of note that association of SSRIs with depression among the offspring was also detected (adjusted HR $=1.84$; 95\%CI: 1.142.97) for preconception exposures (i.e., SSRIs discontinued in pregnancy). In this study, the authors found no association of SSRI in pregnant women with anxiety in children prenatally exposed 35. No other study examined a possible association between antenatal exposure to ADs and psychiatric disorders of later onset (adolescents and young adults) such as schizophrenia 59, and affective and anxiety disorders 60 .

\section{Developmental and cognitive deficits}

Thirty out of 50 studies selected for review focused on the development and/or cognitive deficits in infants, toddlers and preschool children that might have arisen from prenatal exposures to ADs (Table 3). A majority of these 30 studies found no association between prenatal ADs (mostly SSRIs) and deficits of IQ and/or cognitive 61,62,63,64,65,66,67,68,69, language 70, and behavior development 65,71,72,73,74. Some studies, however, indicated that the maternal depressive disorder, regardless of whether it is 
Table 1

Maternal use of antidepressant medication during pregnancy and risk of autism spectrum disorders in children prenatally exposed.

\begin{tabular}{|c|c|c|c|c|c|c|}
\hline $\begin{array}{l}\text { Study (year, } \\
\text { country) }\end{array}$ & Drug exposure & $\begin{array}{c}\text { Design/Sample } \\
\text { characteristics }(\mathbf{N})\end{array}$ & $\begin{array}{l}\text { Main results } \\
\text { [OR, HR or RR } \\
(95 \% \mathrm{Cl})]\end{array}$ & $\begin{array}{l}\text { Strengths/ } \\
\text { Limitations }\end{array}$ & NOS & Conclusion/Remarks \\
\hline $\begin{array}{l}\text { Croen } \\
\text { et al. } 44 \\
(2011, \text { USA) }\end{array}$ & $\begin{array}{l}\text { ADs (before } \\
\text { pregnant 1st, 2nd } \\
\text { \& 3rd trimesters) }\end{array}$ & $\begin{array}{l}\text { Population-based case-control } \\
\text { study. Kaiser Permanent } \\
\text { Medical Care Program in } \\
\text { Northern California (KPNC) } \\
\text { between January } 1995 \text { and } \\
\text { June 1999. Cases ( } \mathrm{n}=298 \\
\text { and their mothers): children } \\
\text { with ASD. Controls ( } \mathrm{n}=1,507 \\
\text { and their mothers): children } \\
\text { without ASD randomly } \\
\text { selected KPNC in January } \\
\text { 1995-November 2002. } \\
\text { Exposure: ADs dispensed at a } \\
\text { KPNC pharmacy }\end{array}$ & $\begin{array}{l}\text { ASD and maternal } \\
\text { use of ADs during } \\
\text { the year prior to } \\
\text { delivery: } 2.2 \text { (1.2- } \\
\text { 4.3\%). ASD and } \\
\text { 1st trimester AD: } \\
\text { 3.8 (1.8-7.8). No } \\
\text { association with } \\
\text { history of mental } \\
\text { illnesses in the } \\
\text { absence of antenatal } \\
\text { exposure to ADs }\end{array}$ & $\begin{array}{l}\text { Data on pharmacy } \\
\text { dispensing may } \\
\text { not reflect actual } \\
\text { use. No control } \\
\text { of confounders } \\
\text { (maternal data/ } \\
\text { lifestyle) }\end{array}$ & 6 & $\begin{array}{l}\text { A 2-fold increase in risk } \\
\text { of ASD associated with } \\
\text { antenatal exposure to } \\
\text { ADs. Risk of ASD was even } \\
\text { higher for exposures to } \\
\text { ADs in the 1st trimester of } \\
\text { pregnancy }\end{array}$ \\
\hline $\begin{array}{l}\text { Hviid et al. } 36 \\
\text { (2013, } \\
\text { Denmark) }\end{array}$ & $\begin{array}{l}\text { SSRI (before } \\
\text { and during } \\
\text { pregnancy) }\end{array}$ & $\begin{array}{l}\text { Population-based cohort } \\
\text { (all 626,875 singleton live } \\
\text { births in Denmark) January } \\
\text { 1996-December 2005, with } \\
\text { follow-up until January 1st, } \\
\text { 2010, or until the child reached } \\
10 \text { years of age, died or was } \\
\text { lost to follow-up, or received } \\
\text { a diagnosis of ASD (Danish } \\
\text { Psychiatric Central Register) }\end{array}$ & $\begin{array}{l}\text { 3,892 ASD cases. No } \\
\text { use of SSRIs (before } \\
\text { and pregnancy) } \\
\text { vs. prenatal SSRI: } \\
\text { fully adjusted RR } \\
=1.20(0.90-1.61) \text {. } \\
\text { No SSRI vs. use of } \\
\text { SSRI before but not } \\
\text { during pregnancy: } \\
\text { fully adjusted RR = } \\
1.46 \text { (1.17-1.81) }\end{array}$ & $\begin{array}{l}\text { Large cohort. } \\
\text { Reduced potential } \\
\text { for selection and } \\
\text { recall biases. Low } \\
\text { prevalence of } \\
\text { pregnancy-related } \\
\text { use of SSRI. No } \\
\text { control of potential } \\
\text { confounders } \\
\text { (mother data/ } \\
\text { lifestyle) }\end{array}$ & 8 & $\begin{array}{l}\text { No significant association } \\
\text { between antenatal } \\
\text { exposure to SSRIs and } \\
\text { increased risk of ASD in } \\
\text { children }\end{array}$ \\
\hline $\begin{array}{l}\text { Sørensen } \\
\text { et al. } 40 \\
\text { ( } 2013 \text {, } \\
\text { Denmark) }\end{array}$ & $\begin{array}{l}\text { ADs (during } \\
\text { pregnancy) }\end{array}$ & $\begin{array}{l}\text { Population-based cohort } \\
\text { of all children born alive } \\
\text { in Denmark ( } \mathrm{n}=668,468 \text { ) } \\
\text { January 1996-December } 2006 \text {. } \\
\text { ASD diagnosis by December } \\
\text { 2010, from Danish Psychiatric } \\
\text { Central Register; ICD-10 code } \\
\text { F84.0, F84.1, F84.5, F84.8, } \\
\text { F84.9). Exposure: women filling } \\
\text { a prescription for ADs } 30 \text { days } \\
\text { before conception to day of } \\
\text { birth. From Danish National } \\
\text { Prescription Registry (January } \\
\text { 1996-December 2006) }\end{array}$ & $\begin{array}{l}\text { Prevalence of } \\
\text { ASD = 1.5\% (5,437 } \\
\text { children), average } \\
\text { age of diagnosis } \\
6.7 \text { years. ASD: } \\
\text { prenatally AD vs. } \\
\text { unexposed: adjusted } \\
\text { HR: } 1.5 \text { (1.2-1.9). } \\
\text { Children born } \\
\text { to mothers with } \\
\text { affective disorders: } \\
\text { AD vs. unexposed; } \\
\text { adjusted HR: } 1.2 \\
\text { (0.7-2.1). AD vs. } \\
\text { unexposed siblings } \\
\text { in families with at } \\
\text { least one child with } \\
\text { ASD: adjusted HR: } \\
0.9 \text { (0.4-2.0) }\end{array}$ & $\begin{array}{l}\text { Large cohort. } \\
\text { Low potential } \\
\text { for selection, } \\
\text { recall and } \\
\text { misclassification } \\
\text { biases. } \\
\text { Prescription } \\
\text { filling data may } \\
\text { not reflect actual } \\
\text { use. No control } \\
\text { of confounding } \\
\text { (mother data/ } \\
\text { lifestyle) }\end{array}$ & 8 & $\begin{array}{l}\text { Association of antenatal } \\
\text { AD with ASD was } \\
\text { not significant when } \\
\text { confounding (maternal } \\
\text { illness, and family-related } \\
\text { factors) was controlled }\end{array}$ \\
\hline
\end{tabular}

(continues) 
Table 1 (continued)

\begin{tabular}{|c|c|c|c|c|c|c|}
\hline $\begin{array}{l}\text { Study (year, } \\
\text { country) }\end{array}$ & Drug exposure & $\begin{array}{c}\text { Design/Sample } \\
\text { characteristics (N) }\end{array}$ & $\begin{array}{l}\text { Main results } \\
\text { [OR, HR or RR } \\
(95 \% \mathrm{CI})]\end{array}$ & $\begin{array}{l}\text { Strengths/ } \\
\text { Limitations }\end{array}$ & NOS & Conclusion/Remarks \\
\hline $\begin{array}{l}\text { Rai et al. } 48 \\
\text { (2013, } \\
\text { Sweden) }\end{array}$ & $\begin{array}{l}\text { SSRI \& non-SSRI } \\
\text { (pregnancy) }\end{array}$ & $\begin{array}{c}\text { Population-based nested } \\
\text { case-control study (Stockholm } \\
\text { cohort, 589,114 people 0-17 } \\
\text { years, 2001-2007). 4,429 } \\
\text { ASD cases (2,601 without } \\
\text { intellectual disability); 43,277 } \\
\text { controls (age and sex- } \\
\text { matched). ASD cases: ICD-9 } \\
\text { (299.0), ICD-10 (F84.0) and } \\
\text { DSM-IV (299). AD use: drug } \\
\text { use data from first antenatal } \\
\text { interview - Swedish Medical } \\
\text { Birth Register }\end{array}$ & $\begin{array}{l}\text { Mother's (but not } \\
\text { father's) depression } \\
\text { associated with } \\
\text { ASD: OR: } 1.49 \text { (1.08- } \\
\text { 2.08). Depression } \\
\text { plus prenatal AD } \\
\text { (SSRI or non-SSRI) } \\
\text { and ASD (adjusted } \\
\text { disorders other than } \\
\text { depression): 3.34 } \\
\text { (1.5-7.47). AD and } \\
\text { ASD: 1.90 (1.15-3.14), } \\
\text { AD and ASD without } \\
\text { intellectual disability: } \\
\text { 2.54 (1.37-4.68) }\end{array}$ & $\begin{array}{c}\text { Mother's } \\
\text { depression } \\
\text { possibly under } \\
\text { ascertained. } \\
\text { Confounding: AD } \\
\text { use may reflect } \\
\text { severe depression } \\
\text { and thus severe } \\
\text { depression (not } \\
\text { AD) would be in } \\
\text { fact associated } \\
\text { with ASD }\end{array}$ & 8 & $\begin{array}{l}\text { Prenatal AD (SSRI \& non- } \\
\text { SSRI) associated with } \\
\text { ASD without intellectual } \\
\text { disability. Association may } \\
\text { be non-causal: additional. } \\
\text { Further research needed } \\
\text { to clarify whether AD } \\
\text { use would reflect severe } \\
\text { maternal depression }\end{array}$ \\
\hline $\begin{array}{l}\text { Rai et al. } 49 \\
\text { (2017, } \\
\text { Sweden) }\end{array}$ & $\begin{array}{l}\text { SSRI \& non-SSRI } \\
\text { (pregnancy) }\end{array}$ & $\begin{array}{l}\text { Prospective cohort study } \\
\text { (Stockholm county, 2001- } \\
\text { 2011, 254,610 individuals } \\
\text { aged 4-17 years, including } \\
\text { 5,378 with ASD). ASD: ICD-9 } \\
\text { (299.0), ICD-10 (F84.0) and } \\
\text { DSM-IV (299). AD use: drug } \\
\text { use data from first antenatal } \\
\text { interview - Swedish Medical } \\
\text { Birth Register. AD use: drug } \\
\text { use data from first antenatal } \\
\text { interview - Swedish Medical } \\
\text { Birth Register }\end{array}$ & $\begin{array}{l}\text { 3,342 children } \\
\text { prenatally exposed } \\
\text { to AD. } 4.1 \% \text { had } \\
\text { ASD compared to } \\
\text { a prevalence of } \\
2.9 \% \text { among those } \\
\text { not exposed to AD } \\
\text { whose mothers had } \\
\text { mental illnesses. } \\
\text { Adjusted OR: } 1.45 \\
\text { (1.13-1.85). Risk } \\
\text { increase concerned } \\
\text { children with ASD } \\
\text { with no intellectual } \\
\text { disability }\end{array}$ & $\begin{array}{l}\text { Large sample size } \\
\text { (total population of } \\
\text { Stockholm county) } \\
\text { and multisource } \\
\text { ascertainment of } \\
\text { cases. Absence } \\
\text { of detailed } \\
\text { measurements } \\
\text { of severity of } \\
\text { depression } \\
\text { in pregnancy. } \\
\text { No trimester } \\
\text { assessment }\end{array}$ & 8 & $\begin{array}{c}\text { Weak (OR = 1.45) } \\
\text { association of ASD in } \\
\text { pregnancy (particularly } \\
\text { ASD without intellectual } \\
\text { disability) with AD (SSRI \& } \\
\text { non-SSRI) }\end{array}$ \\
\hline $\begin{array}{l}\text { El Marroun } \\
\text { et al. } 50 \\
\text { ( } 2014 \text {, } \\
\text { Netherlands) }\end{array}$ & SSRI (pregnancy) & $\begin{array}{l}\text { 5,976 children (study } \\
\text { embedded in the ongoing } \\
\text { population-based cohort: } \\
\text { Generation R Study). Births } \\
\text { from April } 2002 \text { to January } \\
\text { 2006. SSRI exposure: maternal } \\
\text { self-report assessed by } \\
\text { questionnaires, prescription } \\
\text { records from pharmacies. } \\
\text { Parent reported autistic } \\
\text { symptoms (traits) using Child } \\
\text { Behavior Checklists and Social } \\
\text { Responsiveness Scale. }\end{array}$ & $\begin{array}{l}\text { Adjuested (including } \\
\text { adjustment for } \\
\text { postnatal maternal } \\
\text { depression) OR, } \\
\text { pervasive develop. } \\
\text { problems, prenatal } \\
\text { SSRI vs. unexposed: } \\
1.91 \text { (1.13-3.47). } \\
\text { Autistic traits (social } \\
\text { responsiveness } \\
\text { scale) } \beta=0.15, \\
95 \% C l: 0.08-0.22\end{array}$ & $\begin{array}{c}\text { Prospective } \\
\text { study using } 2 \\
\text { measures of } \\
\text { autistic symptoms. } \\
\text { SSRI-treated } \\
\text { women may have } \\
\text { overestimated } \\
\text { problems with } \\
\text { their child. } \\
\text { No clinical } \\
\text { assessments of } \\
\text { ASD. Small number } \\
\text { of SSRI-exposed } \\
\text { children. Residual } \\
\text { confounding } \\
\text { remained }\end{array}$ & 6 & $\begin{array}{c}\text { Results suggested } \\
\text { association between } \\
\text { prenatal SSRI and autistic } \\
\text { traits in children }\end{array}$ \\
\hline
\end{tabular}

(continues) 
Table 1 (continued)

\begin{tabular}{llccc}
\hline $\begin{array}{l}\text { Study (year, Drug exposure } \\
\text { country) }\end{array}$ & $\begin{array}{c}\text { Design/Sample } \\
\text { characteristics (N) }\end{array}$ & $\begin{array}{c}\text { Main results } \\
{[\mathrm{OR}, \mathrm{HR} \text { or RR }} \\
(95 \% \mathrm{CI})]\end{array}$ & $\begin{array}{c}\text { Strengths/ } \\
\text { Limitations }\end{array}$ & NOS Conclusion/Remarks \\
\end{tabular}

\begin{tabular}{|c|c|c|c|c|c|c|}
\hline $\begin{array}{l}\text { Harrington } \\
\text { et al. } 47 \\
(2014, \text { USA) }\end{array}$ & $\begin{array}{l}\text { SSRI (1st, 2nd \& } \\
\text { 3rd trimester) }\end{array}$ & $\begin{array}{l}\text { Population-based case-control } \\
\text { study. CHARGE (Childhood } \\
\text { Autism Risks from Genetics } \\
\text { and Environment). Mother- } \\
\text { child pairs. Children 2-5 } \\
\text { years old from California } \\
\text { with ASD ( } \mathrm{n}=492 \text { ) or DD } \\
\text { other than ASD ( } \mathrm{n}=154) \\
\text { and typical development, } \\
\text { TD (n = 320). Interview } \\
\text { (SSRI use, mental health } \\
\text { history, sociodemographic } \\
\text { information) }\end{array}$ & $\begin{array}{l}\text { OR: among boys }(\widehat{\jmath}) \\
\text { prenatally exposed } \\
\text { to SSRI: ASD vs. TD: } \\
2.91 \text { (1.07-7.93). 1st } \\
\text { trimester: } 3.22(1.17- \\
8.84)\end{array}$ & $\begin{array}{l}\text { Relatively large } \\
\text { sample of cases. } \\
\text { Potential recall } \\
\text { bias. Residual } \\
\text { confounding by } \\
\text { indication of SSRI, } \\
\text { no assessment } \\
\text { of depression } \\
\text { severity. No data } \\
\text { on SSRI dosage }\end{array}$ & 8 & $\begin{array}{c}\text { In boys, prenatal exposure } \\
\text { to SSRI increases risk of } \\
\text { ASD }\end{array}$ \\
\hline $\begin{array}{l}\text { Gidaya et } \\
\text { al. } 45 \\
\text { (2014, } \\
\text { Denmark) }\end{array}$ & $\begin{array}{l}\text { SSRI (pre- } \\
\text { conception, } \\
\text { 1st, 2nd \& 3rd } \\
\text { trimesters) }\end{array}$ & $\begin{array}{l}\text { Population-based (Danish } \\
\text { Civil Registration System) } \\
\text { case-control study. All } \\
\text { children (singleton or one } \\
\text { selected at random from } \\
\text { multiple births) born alive } \\
\text { in Denmark ( } \mathrm{n}=628,408 \text { ) } \\
\text { Januarty 1997-December } \\
\text { 2006. Cases: ASD diagnosis } \\
\text { (Danish National Hospital } \\
\text { Register, January 1999-March } \\
\text { 2011). Controls without } \\
\text { ASD. } 10 \text { per case. Exposure: } \\
\text { dispensed. From: Danish Drug } \\
\text { Prescription Registry }\end{array}$ & $\begin{array}{l}\text { 1.5\% of cases and } \\
0.7 \% \text { of controls } \\
\text { exposed to SSRI } \\
\text { during pregnancy. } \\
\text { Adjusted OR, ASD } \\
\text { vs. maternal SSRI } \\
\text { use (> } 45 \text { days); } \\
\text { preconception; } \\
2.1 \text { (1.6-2.8); } 1 \text { st } \\
\text { trimester; } 2.4 \text { (1.7- } \\
\text { 3.3); 2nd trimester; } \\
\text { 2.4 (1.6-3.5); 3rd } \\
\text { trimester; } 2.9 \\
\text { (1.9-4.4). Adjusted } \\
\text { for: age, child sex, } \\
\text { mother depression; } \\
\text { other SSRI } \\
\text { indications }\end{array}$ & $\begin{array}{l}\text { Large population- } \\
\text { based case-control } \\
\text { study. Residual } \\
\text { confounding } \\
\text { by indication of } \\
\text { SSRI and severity } \\
\text { of maternal } \\
\text { depression }\end{array}$ & 8 & $\begin{array}{l}\text { Study results suggest that } \\
\text { antenatal exposure to } \\
\text { SSRI increases child's risk } \\
\text { associated with ASD }\end{array}$ \\
\hline $\begin{array}{l}\text { Clements } \\
\text { et al. } 41 \\
(2015, \text { USA) }\end{array}$ & $\begin{array}{l}\text { SSRI/non-SSRI } \\
\text { (pre-conception, } \\
\text { 1st, 2nd \& 3rd } \\
\text { trimesters) }\end{array}$ & $\begin{array}{l}\text { Case-control study. Cases: } \\
\text { children from The Partners } \\
\text { HealthCare EHR (New England, } \\
\text { Boston, Massachusetts) } \\
\text { with 2-19 years, if they had } \\
\text { at least one ICD-9 code } \\
\text { of 299, 1997-2000. Cases: } \\
\text { 1,377 children with ASD; } \\
\text { Controls: no prior history of } \\
\text { ASD, ADHD or intellectual } \\
\text { disability; matched (1:3) for } \\
\text { birth year, birth hospital, } \\
\text { sex, insurance type (proxy } \\
\text { for socioeconomic status), } \\
\text { ethnicity, preterm vs. full term. } \\
\text { AD prescription (outpatient } \\
\text { EHR) and dispensing (inpatient } \\
\text { pharmacy) }\end{array}$ & $\begin{array}{c}\text { Adjusted OR; } \\
\text { pre-pregnancy } \\
\text { (adjusted mother's } \\
\text { depression) } \\
1.62 \text { (1.17-2.23). } \\
\text { Pregnancy (pre- } \\
\text { pregnancy-delivery), } \\
\text { non-adjusted } \\
\text { 1.49 (1.01-2.18), } \\
\text { adjuested 1.10 (0.70- } \\
\text { 1.70), 1st trimester } \\
\text { 1.47 (0.81-2.61), 2nd } \\
\text { trimester (1.34 (0.77- } \\
\text { 2.27), 3rd trimester } \\
\text { 1.08 (0.61-1.88) }\end{array}$ & $\begin{array}{l}\text { Relatively large } \\
\text { sample of cases. } \\
\text { Confounding } \\
\text { by indication, } \\
\text { misclassification } \\
\text { bias }\end{array}$ & 7 & $\begin{array}{l}\text { ASD risk associated with } \\
\text { prenatal ADs was no longer } \\
\text { significant after controlling } \\
\text { for maternal depression }\end{array}$ \\
\hline
\end{tabular}

(continues) 
Table 1 (continued)

\begin{tabular}{|c|c|c|c|c|c|c|}
\hline $\begin{array}{l}\text { Study (year, } \\
\text { country) }\end{array}$ & Drug exposure & $\begin{array}{c}\text { Design/Sample } \\
\text { characteristics (N) }\end{array}$ & $\begin{array}{l}\text { Main results } \\
\text { [OR, HR or RR } \\
(95 \% \mathrm{Cl})]\end{array}$ & $\begin{array}{l}\text { Strengths/ } \\
\text { Limitations }\end{array}$ & NOS & Conclusion/Remarks \\
\hline $\begin{array}{l}\text { Boukhris } \\
\text { et al. } 46 \\
\text { (2016, } \\
\text { Canada) }\end{array}$ & $\begin{array}{c}\text { ADs (1st, 2nd \& 3rd } \\
\text { trimesters) }\end{array}$ & $\begin{array}{l}\text { Population-based cohort, } \\
\text { January 1998-December } 2009 . \\
\text { 145,456 full-term singleton } \\
\text { infants born alive. ASD: } \\
\text { children with at least } 1 \text { ASD } \\
\text { diagnosis between date of } \\
\text { birth and last follow-up }\end{array}$ & $\begin{array}{c}\text { 1,054 (0.7\%, 4 }: 1 \text { }) \\
\text { with ASD. Age of } \\
\text { children at the end } \\
\text { of follow-up was } \\
6.24 \text { (3.19) years. } \\
\text { 2nd/3rd trimester } \\
\text { adjuested HR; ADs } \\
=1.87 \text { (1.15-3.04), } \\
\text { SSRIs = 2.17 (1.20- } \\
\text { 3.93). Adjuested } \\
\text { for maternal } \\
\text { depression: ADs = } \\
\text { 1.75 (1.03-2.97) }\end{array}$ & $\begin{array}{l}\text { Large cohort, } \\
\text { 11-year follow- } \\
\text { up. Prescription } \\
\text { filling data (may } \\
\text { not reflect actual } \\
\text { use), lack of } \\
\text { control of potential } \\
\text { confounders } \\
\text { (mother data/ } \\
\text { lifestyle) }\end{array}$ & 8 & $\begin{array}{c}\text { Antenatal ADs, } \\
\text { (predominantly SSRIs), } \\
\text { in the 2nd/3rd trimester } \\
\text { increases ASD risk }\end{array}$ \\
\hline $\begin{array}{l}\text { Castro } \\
\text { et al. } 37 \\
(2016, \text { USA })\end{array}$ & $\begin{array}{l}\text { SSRI/non-SSRI } \\
\text { (pre-pregnancy, } \\
\text { 1st, 2nd \& 3rd } \\
\text { trimester) }\end{array}$ & $\begin{array}{l}\text { Case-control study. Cases: } \\
\text { children from The Partners } \\
\text { HealthCare EHR with 2-19 } \\
\text { years, at least one ICD-9 code } \\
\text { of 299. 1997-2010. Cases: } \\
\text { 1,245. ASD; Controls: no } \\
\text { prior history of ASD, ADHD } \\
\text { or intellectual disability; } \\
\text { matched (1:3) for birth year, } \\
\text { birth hospital, sex, insurance } \\
\text { type (proxy for socioeconomic } \\
\text { status), ethnicity, preterm vs } \\
\text { full term. Mother-child pairs } \\
\text { included in the previous } \\
\text { report by Clements et } \\
\text { al. } 41 \text { were excluded. AD } \\
\text { prescription (outpatient EHR) } \\
\text { and dispensing (inpatient } \\
\text { pharmacy) }\end{array}$ & $\begin{array}{c}\text { Adjusted OR; pre- } \\
\text { pregnancy (adjusted } \\
\text { for maternal } \\
\text { depression) } \\
1.54(1.02-2.30) . \\
\text { Pregnancy (pre- } \\
\text { pregnancy-delivery), } \\
\text { non-adjusted 0.99 } \\
\text { (0.63-1.51), adjusted } \\
0.90(0.50-1.54), 1 \text { st } \\
\text { trimester } 0.89(0.40- \\
1.78), 2 \text { nd trimester } \\
(1.11(0.50-2.26), 3 \text { rd } \\
\text { trimester } 0.85(0.38- \\
1.74)\end{array}$ & $\begin{array}{l}\text { Relatively large } \\
\text { sample of cases. } \\
\text { Some risk of } \\
\text { confounding } \\
\text { by indication, } \\
\text { misclassification } \\
\text { bias }\end{array}$ & 7 & $\begin{array}{l}\text { Pre-pregnancy only use of } \\
\text { ADs associated with ASD. } \\
\text { No association between } \\
\text { mother use of AD in } \\
\text { pregnancy and ASD }\end{array}$ \\
\hline $\begin{array}{l}\text { Sujan et al. } 42 \\
(2017, \\
\text { Sweden })\end{array}$ & $\begin{array}{l}\text { ADs (1st } \\
\text { trimmester) }\end{array}$ & $\begin{array}{l}\text { Retrospective cohort study: } \\
\text { Swedish offspring born 1996- } \\
2012 \text { followed through 2013, } \\
\text { death or emigration. Maternal } \\
\text { self-reported 1st trimester use } \\
\text { and dispensation of ADs; data } \\
\text { from Medical Birth Register, } \\
\text { and Prescribed Drug Register. } \\
\text { ASD inpatient and outpatient } \\
\text { diagnoses made by a specialist } \\
\text { according to ICD-9 and ICD-10 } \\
\text { criteria (Swedish registers) }\end{array}$ & $\begin{array}{l}\text { Analytical cohort } \\
\text { (1,580,629 children, } \\
48.6 \% \text { females), } \\
\text { 1.4\% were exposed } \\
\text { (mother self-report) } \\
\text { to ADs during } 1 \text { st } \\
\text { trimester and of } \\
\text { these, } 82 \% \text { were } \\
\text { exposed to SSRIs. } \\
\text { ASD exposed } \\
\text { vs. unexposed; } \\
\text { HR: } 2.0 \text { (1.8-2.3). } \\
\text { Siblings analysis } \\
\text { adjusted pregnancy, } \\
\text { maternal, paternal } \\
\text { traits; ASD; } 0.8 \text { (0.6- } \\
1.1 \text { ) }\end{array}$ & $\begin{array}{l}\text { Large population } \\
\text { based sample } \\
\text { (cohort study), } \\
\text { Sibling analysis. } \\
\text { Exposure } \\
\text { conferred both } \\
\text { to self-report and } \\
\text { dispensation. } \\
\text { Recall bias. No } \\
\text { assessment } \\
\text { of maternal } \\
\text { depression } \\
\text { severity. 1st } \\
\text { trimester exposure } \\
\text { only. Mostly SSRIs }\end{array}$ & 8 & $\begin{array}{l}\text { After taking into account } \\
\text { confounding; no } \\
\text { association of 1st trimester } \\
\text { exposure to ADs with ASD }\end{array}$ \\
\hline
\end{tabular}

(continues) 
Table 1 (continued)

\begin{tabular}{|c|c|c|c|c|c|c|}
\hline $\begin{array}{l}\text { Study (year, } \\
\text { country) }\end{array}$ & Drug exposure & $\begin{array}{c}\text { Design/Sample } \\
\text { characteristics (N) }\end{array}$ & $\begin{array}{l}\text { Main results } \\
\text { [OR, HR or RR } \\
(95 \% \mathrm{CI})]\end{array}$ & $\begin{array}{l}\text { Strengths/ } \\
\text { Limitations }\end{array}$ & NOS & Conclusion/Remarks \\
\hline $\begin{array}{l}\text { Malm et al. } 35 \\
(2016, \\
\text { Finland) }\end{array}$ & SSRI (pregnancy) & $\begin{array}{l}\text { Population-based cohort } \\
\text { study. Data from Finnish } \\
\text { National Registers. 845,345 } \\
\text { singleton live births in } \\
\text { Finland between January } \\
\text { 1996-December 2010. Age } \\
\text { range from birth to age 14, } \\
\text { mother child pairs - National } \\
\text { Medical Birth Register. Drug } \\
\text { Reimbursement Register } \\
\text { (prescription drug purchases). } \\
\text { Outcome: ASD }\end{array}$ & $\begin{array}{c}\text { ASD, adjusted HR; } \\
\text { SSRI gestation vs. } \\
\text { Mother psychiatric } \\
\text { disease and no } \\
\text { medication, } 0.88 \\
\text { (0.65-1.20), SSRI } \\
\text { pregnancy vs. SSRI } \\
\text { pre-pregnancy only, } \\
\text { 1.30 (0.88-1.92), SSRI } \\
\text { pregnancy vs. no } \\
\text { maternal disease, } \\
\text { no medication, 1.40 } \\
\text { (1.02-1.92) }\end{array}$ & $\begin{array}{c}\text { Large population } \\
\text { based prospective } \\
\text { cohort. No } \\
\text { pregnancy } \\
\text { trimester analysis. } \\
\text { No confirmation } \\
\text { of adherence to } \\
\text { purchased drugs. } \\
\text { No information } \\
\text { on postnatal } \\
\text { environment }\end{array}$ & 7 & $\begin{array}{c}\text { Taking into account } \\
\text { maternal illness, prenatal } \\
\text { SSRI was not associated } \\
\text { with increased risk of ASD. }\end{array}$ \\
\hline $\begin{array}{l}\text { Brown } \\
\text { et al. } 38 \\
\text { (2017, } \\
\text { Canada) }\end{array}$ & $\begin{array}{l}\text { SSRI/SNRI (during } \\
\text { pregnancy) }\end{array}$ & $\begin{array}{l}\text { Retrospective cohort study } \\
\text { using health administrative } \\
\text { data from Ontario, Canada, } \\
\text { children ( } 35,906 \text { singleton } \\
\text { births) born to mothers } \\
\text { receiving public prescription } \\
\text { drug (4.2\% births) from } 2002- \\
2010 . \text { Outcome followed } \\
\text { through 2014. ASD diagnosed } \\
\text { after the age of } 2 \text { years }\end{array}$ & $\begin{array}{c}\text { ASD adjusted HR; } \\
\text { SSRI vs. unexposed } \\
1.59 \text { (1.17-2.17), } \\
\text { sibling analysis, } 1.60 \\
(0.69-3.74)\end{array}$ & $\begin{array}{l}\text { No remarkable } \\
\text { strength limited } \\
\text { to women in } \\
\text { Ontario's publicly } \\
\text { funded drug } \\
\text { plan (lower social } \\
\text { class). Potential } \\
\text { misclassification } \\
\text { bias (filled-out } \\
\text { prescriptions not } \\
\text { used) }\end{array}$ & 8 & $\begin{array}{l}\text { Prenatal exposure to SSRI } \\
\text { or SNRI was not associated } \\
\text { to ASD in children }\end{array}$ \\
\hline $\begin{array}{l}\text { Viktorin } \\
\text { et al. } 39 \\
\text { (2017, } \\
\text { Sweden) }\end{array}$ & ADs (pregnancy) & $\begin{array}{l}\text { Population based cohort of } \\
\text { 179,007 Swedish children } \\
\text { born in 2006-2007 followed } \\
\text { through } 2014 \text { when they } \\
\text { were aged } 7 \text { and 8. Mother } \\
\text { and children identified in } \\
\text { the Swedish Medical Birth } \\
\text { Register. Exposure: data on } \\
\text { prescription drug dispensed } \\
\text { - Swedish Drug Register, ASD; } \\
\text { ICD-10 codes F84.0, F84.1, } \\
\text { F84.2, F84.3, F84.4, F84.5, } \\
\text { F84.8, F84.9 - Swedish Patient } \\
\text { Register }\end{array}$ & $\begin{array}{l}\text { ASD adjusted RR } \\
\text { (95\%Cl), full sample, } \\
\text { ADs vs. unexposed: } \\
1.23 \text { (0.96-1.57). } \\
\text { Subsample of } \\
\text { mothers with at } \\
\text { least one diagnosis } \\
\text { of depression or } \\
\text { anxiety in their } \\
\text { lifetime; ADs ( } 2 \\
\text { dispensations } \\
\text { overlapping } \\
\text { pregnancy) vs. } \\
\text { unexposed: } \\
1.07 \text { (0.80-1.43). } \\
\text { citalopram/ } \\
\text { escitalopram: } \\
1.47 \text { (0.92-2.35), } \\
\text { clomipramine: } 2.86 \\
\text { (1.04-7.82) }\end{array}$ & $\begin{array}{l}\text { Nationwide } \\
\text { population-based } \\
\text { cohort. Severity of } \\
\text { maternal mental } \\
\text { illness not known }\end{array}$ & 8 & $\begin{array}{c}\text { Prenatal exposure to ADs } \\
\text { was not causally associated } \\
\text { with ASD in children }\end{array}$ \\
\hline
\end{tabular}

(continues) 
Table 1 (continued)

\begin{tabular}{|c|c|c|c|c|c|c|}
\hline $\begin{array}{l}\text { Study (year, } \\
\text { country) }\end{array}$ & Drug exposure & $\begin{array}{c}\text { Design/Sample } \\
\text { characteristics (N) }\end{array}$ & $\begin{array}{l}\text { Main results } \\
\text { [OR, HR or RR } \\
\quad(95 \% \mathrm{CI})]\end{array}$ & $\begin{array}{l}\text { Strengths/ } \\
\text { Limitations }\end{array}$ & NOS & Conclusion/Remarks \\
\hline $\begin{array}{l}\text { Hagberg } \\
\text { et al. } 43 \\
(2018, \text { UK) }\end{array}$ & $\begin{array}{l}\text { ADs (pre- } \\
\text { pregnancy, } \\
\text { 1st, 2nd \& 3rd } \\
\text { trimester) }\end{array}$ & $\begin{array}{l}\text { Cohort study (with nested } \\
\text { sibling case-control analysis) } \\
\text { using a population-based } \\
\text { electronic medical database } \\
\text { (UK Medical Practice Research } \\
\text { Datalink, CPRD). Mothers } \\
\text { (aged } 13 \text { to } 44 \text { years) and } \\
\text { their live born singleton } \\
\text { infants, born between } 1989 \\
\text { and 2011. Cohort entry was } \\
\text { baby delivery date minus } \\
365 \text { days. Children: at least } 3 \\
\text { years of follow-up after birth. } \\
3 \text { cohorts of exposed women } \\
\text { (depressed treated with AD); } \\
\text { depressed untreated with AD } \\
\text { in pregnancy; non-depressed } \\
\text { treated with AD in pregnancy). } \\
\text { 194,494 mother-baby pairs; } \\
\text { 2,154 with ASD }\end{array}$ & $\begin{array}{c}\text { RR; unexposed vs. } \\
\text { treated depression: } \\
1.72 \text { (1.54-1.93); } \\
\text { unexposed } \\
\text { vs. untreated } \\
\text { depression: } \\
1.50 \text { (1.28-1.75); } \\
\text { unexposed vs. AD } \\
\text { for disorders other } \\
\text { than depression: } \\
0.73 \text { (0.41-1.29). } \\
\text { Sibling analysis yield } \\
\text { results similar to } \\
\text { those of main study. } \\
\text { Additional analysis } \\
\text { indicated that risk of } \\
\text { ASD increased with } \\
\text { increasing severity } \\
\text { of depression } \\
\text { (duration of last } \\
\text { episode of untreated } \\
\text { depression; }<12 \\
\text { months vs. } 12-35.9 \\
\text { months) }\end{array}$ & $\begin{array}{l}\text { Large population- } \\
\text { based cohorts. } \\
\text { Assessment } \\
\text { of effects of } \\
\text { untreated } \\
\text { depression } \\
\text { and severity } \\
\text { of untreated } \\
\text { depression. } \\
\text { No remarkable } \\
\text { weakness }\end{array}$ & 9 & $\begin{array}{l}\text { Pregnant women with } \\
\text { depression have increased } \\
\text { risk of having a child with } \\
\text { ASD, whether or not she } \\
\text { used ADs during pregnancy }\end{array}$ \\
\hline
\end{tabular}

95\%Cl: 95\% confidence interval; ADs: antidepressants; ASD: autism spectrum disorders; DD: developmental delays; DSM-IV: Diagnostic and Statistical Manual of Mental Disorders - 4th edition; EHR: electronic health record; HR: hazard ratio; ICD: International Classification of Diseases; NOS: Newcastle-Ottawa Scale; OR: odds ratio; RR: rate ratio; SNRI: serotonin-norepinephrine reuptake inhibitors;

SSRI: selective serotonin reuptake inhibitors; TD: traditional development. 
Table 2

Maternal use of antidepressant medications in pregnancy and risk of attention deficit/hyperactivity disorders in prenatally exposed children.

\begin{tabular}{|c|c|c|c|c|c|c|}
\hline $\begin{array}{l}\text { Study (year, } \\
\text { country) }\end{array}$ & Drug exposure & $\begin{array}{c}\text { Design/Sample } \\
\text { characteristics (N) }\end{array}$ & $\begin{array}{c}\text { Main results } \\
\text { [OR or HR }(95 \% \mathrm{CI})]\end{array}$ & $\begin{array}{l}\text { Strengths/ } \\
\text { Limitations }\end{array}$ & NOS & Conclusion/Remarks \\
\hline $\begin{array}{l}\text { Figueroa } 52 \\
(2010, \text { USA) }\end{array}$ & $\begin{array}{l}\text { ADs (1st, 2nd \& 3rd } \\
\text { trimesters) }\end{array}$ & $\begin{array}{l}\text { Retrospective cohort } \\
\text { study. Claims data (self- } \\
\text { insured employers) on } \\
\text { 38,074 children born } \\
\text { 1997-2002 and their } \\
\text { families until they were } \\
4 \text { years old (1997-2006). } \\
\text { Data on in/outpatient } \\
\text { prescription claims; } \\
\text { diagnoses and others. } \\
\text { Outcome: ADHD at the } \\
\text { age of } 5 \text { or earlier }\end{array}$ & $\begin{array}{l}431 \text { children diagnosed } \\
\text { or treated for ADHD } \leq 5 \\
\text { years. SSRI in pregnancy } \\
\text { OR }=0.91(0.51-1.60) ; \\
\text { BUP in pregnancy } 3.63 \\
(1.20-11.04) ; 1 \text { st trimester } \\
2.06(0.35-12.16), 2 \text { nd } \\
\text { trimester } 14.66 \text { (3.27- } \\
65.73) ; \text { other ADs in } \\
\text { pregnancy: } 0.65 \text { (0.09- } \\
4.79)\end{array}$ & $\begin{array}{l}\text { ADHD typically } \\
\text { diagnosed in } \\
\text { children > } 5 \text { years. } \\
\text { Prescription data } \\
\text { may not reflect } \\
\text { actual use. Failure to } \\
\text { control confounding } \\
\text { (mother's disease } \\
\text { and lifestyle) }\end{array}$ & 7 & $\begin{array}{l}\text { Prenatal SSRIs and ADs } \\
\text { other than BUP not } \\
\text { associated with ADHD in } \\
\text { children at the age of } 5 \\
\text { or younger. Bupropion, } \\
\text { especially in the } 2 \text { nd } \\
\text { trimester, increased risks } \\
\text { of ADHD }\end{array}$ \\
\hline $\begin{array}{l}\text { Laugesen et al. } 51 \\
\text { (2013, Denmark) }\end{array}$ & $\begin{array}{l}\text { ADs (1 } 1 \text { st, } 2^{\text {nd }} \& 3^{\text {rd }} \\
\text { trimesters })\end{array}$ & $\begin{array}{l}\text { Cohort of all singletons } \\
\text { born alive (N = 877,778) } \\
\text { in Denmark (Danish } \\
\text { Medical Birth Registry) } \\
\text { from } 1996 \text { until the } \\
\text { end of } 2009 \text {. Exposure: } \\
\text { maternal redemption } \\
\text { of an AD prescription } \\
\text { through the Danish } \\
\text { National Prescription } \\
\text { Registry. Outcome: } \\
\text { diagnosis of ADHD or } \\
\text { prescription for ADHD } \\
\text { medication (Danish } \\
\text { Psychiatric Registry, } \\
\text { Danish National Registry } \\
\text { of Patients. Overall } \\
\text { median follow up time = } \\
8 \text { years }\end{array}$ & $\begin{array}{c}\text { 15,008 (1.7\%) were } \\
\text { exposed to AD. 12,841 } \\
\text { (1.5\%) developed ADHD. } \\
\text { SSRI the most used (78\% } \\
\text { of AD users). Adjusted } \\
\text { HR any AD pregnancy } 1.2 \\
\text { (1.1-1.4), 1st trimester } 1.2 \\
\text { (1.0-1.4), 2nd trimester } \\
\text { 1.5 (0.9-2.4), 3rd trimester } \\
0.8 \text { (0.3-2.0), pregnancy } \\
\text { use: SSRI, 1.2 (1.0-1.5), } \\
\text { SNRI 1.0 (0.4-2.5), TCA } \\
\text { 1.1 (0.6-20), others 1.6 } \\
\text { (0.8-3.0). Sibling analysis: } \\
\text { 0.7 (0.4-1.4). Former } \\
\text { users vs. never users: } 1.6 \\
\text { (1.5-1.8) }\end{array}$ & $\begin{array}{l}\text { Large population- } \\
\text { based cohort. No } \\
\text { data on actual AD } \\
\text { intake by the mother } \\
\text { (prescription is a } \\
\text { proxy) }\end{array}$ & 8 & $\begin{array}{l}\text { Long-term follow-up: no } \\
\text { evidence of association } \\
\text { of prenatal AD with } \\
\text { ADHD }\end{array}$ \\
\hline $\begin{array}{l}\text { Clements et al. } 41 \\
(2015, \text { USA) }\end{array}$ & $\begin{array}{l}\text { SSRI/non-SSRI } \\
\text { (pre-pregnancy, } \\
\text { 1st, 2nd \& 3rd } \\
\text { trimesters) }\end{array}$ & $\begin{array}{l}\text { Case-control study. } \\
\text { Children from The } \\
\text { Partners HealthCare EHR } \\
\text { (Boston, Massachusetts) } \\
\text { with 2-19 years. Cases: } \\
\text { 2,243 children with } \\
\text { ADHD (but no ASD); } \\
\text { Controls (5,631): no prior } \\
\text { history of ADHD, ASD } \\
\text { or intellectual disability; } \\
\text { matched (1:3) for birth } \\
\text { year, birth hospital, sex, } \\
\text { insurance type (proxy } \\
\text { for socioeconomic } \\
\text { status), ethnicity, pre- } \\
\text { term vs. full term. AD } \\
\text { prescription (outpatient } \\
\text { EHR) and dispensing } \\
\text { (inpatient pharmacy) }\end{array}$ & $\begin{array}{c}\text { Adjusted OR; pre- } \\
\text { pregnancy (adjusted } \\
\text { history of maternal } \\
\text { depression) } 1.18 \text { (0.86- } \\
\text { 1.61). Pregnancy (pre- } \\
\text { pregnancy-delivery), } \\
\text { non-adjusted 2.30 } \\
\text { (1.62-3.24), adjusted } 1.81 \\
(1.22-2.70), 1 \text { st trimester } \\
2.03 \text { (1.19-3.44), 2nd } \\
\text { trimester 0.98 (0.56- } \\
1.68), 3 \text { rd trimester } 1.29 \\
(0.76-2.15)\end{array}$ & $\begin{array}{l}\text { Relatively large } \\
\text { sample of cases. } \\
\text { Confounding } \\
\text { by indication, } \\
\text { misclassification bias }\end{array}$ & 7 & $\begin{array}{c}\text { Prenatal ADs was } \\
\text { associated with a } \\
\text { modest increase in risk } \\
\text { of ADHD even after } \\
\text { adjustment to maternal } \\
\text { depression }\end{array}$ \\
\hline
\end{tabular}

(continues) 
Table 2 (continued)

\begin{tabular}{|c|c|c|c|c|c|c|}
\hline $\begin{array}{l}\text { Study (year, } \\
\text { country) }\end{array}$ & Drug exposure & $\begin{array}{c}\text { Design/Sample } \\
\text { characteristics (N) }\end{array}$ & $\begin{array}{c}\text { Main results } \\
\text { [OR or HR }(95 \% \mathrm{Cl})]\end{array}$ & $\begin{array}{l}\text { Strengths/ } \\
\text { Limitations }\end{array}$ & NOS & Conclusion/Remarks \\
\hline $\begin{array}{l}\text { Castro et al. } 37 \\
(2016, \text { USA) }\end{array}$ & $\begin{array}{l}\text { AD (SSRI/ } \\
\text { non-SSRI) (pre- } \\
\text { pregnancy, } \\
\text { 1st, 2nd \& 3rd } \\
\text { trimesters) }\end{array}$ & $\begin{array}{l}\text { Case-control study. } \\
\text { Children from The } \\
\text { Partners HealthCare } \\
\text { EHR with 2-19 years. } \\
\text { Cases: 1,701 ADHD; } \\
\text { Controls: no prior } \\
\text { history of ADHD, ASD, } \\
\text { or intellectual disability; } \\
\text { matched (1:3) for birth } \\
\text { year, birth hospital, sex, } \\
\text { insurance type (proxy } \\
\text { for socioeconomic } \\
\text { status), ethnicity, pre- } \\
\text { term vs. full term. AD } \\
\text { prescription (outpatient } \\
\text { EHR) and dispensing } \\
\text { (inpatient pharmacy) }\end{array}$ & $\begin{array}{c}\text { Adjusted OR pre- } \\
\text { pregnancy (adjusted } \\
\text { history of maternal } \\
\text { depression) } 1.50 \text { (1.00- } \\
\text { 2.20). Pregnancy (pre- } \\
\text { pregnancy-delivery), } \\
\text { non-adjusted } 0.91 \\
(0.56-1.42) \text {, adjusted } 0.97 \\
(0.53-1.69), 1 \text { st trimester } \\
0.69 \text { (0.29-1.49), 2nd } \\
\text { trimester } 1.11(0.50- \\
2.26), 3 \text { rd trimester } 0.73 \\
(0.29-1.62)\end{array}$ & $\begin{array}{l}\text { Relatively large } \\
\text { sample of cases. } \\
\text { Risk of confounding } \\
\text { by indication, } \\
\text { misclassification bias }\end{array}$ & 7 & $\begin{array}{l}\text { Pre-pregnancy use of } \\
\text { ADs modestly associated } \\
\text { with } A D H D \text { in children. } \\
\text { No association between } \\
\text { maternal use of AD in } \\
\text { pregnancy and ADHD in } \\
\text { the offspring }\end{array}$ \\
\hline $\begin{array}{l}\text { Malm et al. } 35 \\
(2016, \text { Finland) }\end{array}$ & $\begin{array}{l}\text { SSRI (during } \\
\text { pregnancy) }\end{array}$ & $\begin{array}{l}\text { Population-based cohort } \\
\text { study. Data from Finnish } \\
\text { National Registers. } \\
845,345 \text { singleton live } \\
\text { births between January } \\
\text { 1996-December } 2010 . \\
\text { Age range: birth to age } \\
\text { 14, mother child pairs - } \\
\text { National Medical Birth } \\
\text { Register. ICD-8, ICD-9 } \\
\text { and ICD-10 (since 1996) } \\
\text { Drug Reimbursement } \\
\text { Register (prescription } \\
\text { drug purchases). } \\
\text { Outcome: ADHD }\end{array}$ & $\begin{array}{l}\text { ADHD, adjusted HR } \\
(95 \% \mathrm{Cl}) \text {; SSRI gestation } \\
\text { vs. Mother psychiatric } \\
\text { disease and no } \\
\text { medication, } 0.98 \text { (0.77- } \\
\text { 1.24), SSRI pregnancy } \\
\text { vs. SSRI pre-pregnancy } \\
\text { only, } 0.98 \text { (0.75-1.27), } \\
\text { SSRI pregnancy vs. no } \\
\text { maternal disease, no } \\
\text { medication, 1.66 (1.27- } \\
2.16)\end{array}$ & $\begin{array}{l}\text { Large population } \\
\text { based prospective } \\
\text { cohort. No pregnancy } \\
\text { trimester analysis. } \\
\text { No confirmation of } \\
\text { drug adherence. No } \\
\text { data on postnatal } \\
\text { environment }\end{array}$ & 8 & $\begin{array}{l}\text { Taking into account } \\
\text { maternal disorder, } \\
\text { prenatal SSRI was not } \\
\text { associated with ADHD in } \\
\text { children }\end{array}$ \\
\hline $\begin{array}{l}\text { Sujan et al. }{ }^{42} \\
\text { (2017, Sweden) }\end{array}$ & $\begin{array}{l}\text { ADs (pregnancy } \\
\text { 1st trimester) }\end{array}$ & $\begin{array}{l}\text { Retrospective cohort } \\
\text { study Swedish offspring } \\
\text { born 1996-2012 } \\
\text { followed through } 2013 \text {, } \\
\text { death or emigration. } \\
\text { Maternal self-reported } \\
\text { 1st trimester use and } \\
\text { dispensation of ADs; } \\
\text { data from Medical Birth } \\
\text { Register, and prescribed } \\
\text { Drug Register. ADHD, } \\
\text { inpatient and outpatient } \\
\text { diagnoses made by } \\
\text { specialist according to } \\
\text { ICD-9 and ICD-10 criteria } \\
\text { (Swedish registers) }\end{array}$ & $\begin{array}{l}\text { Analytical cohort } \\
\text { (1,580,629 children, } \\
\text { 48.6\% females), } 1.4 \% \\
\text { were exposed (maternal } \\
\text { self-report) to ADs } \\
\text { during } 1 \text { st trimester } \\
\text { and of these, } 82 \% \text { were } \\
\text { exposed to SSRIs. ADHD } \\
\text { exposed vs unexposed; } \\
\text { HR ( } 95 \% \text { CI): } 2.2 \text { (2.0-2.4). } \\
\text { Comparison of siblings, } \\
\text { adjusted pregnancy, } \\
\text { maternal, paternal traits; } \\
\text { ADHD; } 1.0 \text { (0.8-1.3) }\end{array}$ & $\begin{array}{l}\text { Large population } \\
\text { based sample } \\
\text { (cohort study), } \\
\text { Sibling comparison. } \\
\text { Exposure conferred } \\
\text { both to self-report } \\
\text { and dispensation. } \\
\text { Recall bias. Register- } \\
\text { based approach did } \\
\text { not assess maternal } \\
\text { depression and its } \\
\text { severity. 1st trimester } \\
\text { exposure only. } \\
\text { Mostly SSRIs }\end{array}$ & 8 & $\begin{array}{l}\text { After accounting for } \\
\text { confounding factors; } \\
\text { no association of } 1 \text { st } \\
\text { trimester exposure } \\
\text { to ADs with ADHD in } \\
\text { children }\end{array}$ \\
\hline
\end{tabular}

(continues) 
Table 2 (continued)

\begin{tabular}{|c|c|c|c|c|c|c|}
\hline $\begin{array}{l}\text { Study (year, } \\
\text { country) }\end{array}$ & Drug exposure & $\begin{array}{c}\text { Design/Sample } \\
\text { characteristics (N) }\end{array}$ & $\begin{array}{c}\text { Main results } \\
\text { [OR or HR }(95 \% \mathrm{CI})]\end{array}$ & $\begin{array}{l}\text { Strengths/ } \\
\text { Limitations }\end{array}$ & NOS & Conclusion/Remarks \\
\hline $\begin{array}{l}\text { Man et al. } 58 \\
(2017, \text { China) }\end{array}$ & $\begin{array}{c}\text { ADs (1 st, 2nd \& 3rd } \\
\text { trimesters) }\end{array}$ & $\begin{array}{l}\text { Population-based } \\
\text { cohort study (Hong } \\
\text { Kong territory wide) } \\
\text { nested in Clinical Data } \\
\text { Analysis and Reporting } \\
\text { System (CDARS). All live } \\
\text { born children in Hong } \\
\text { Kong from January } \\
2001 \text { to December } \\
2009 \text { (n = 190,618). } \\
\text { Exposure: maternal } \\
\text { ADs prescribing and } \\
\text { dispensing records } \\
\text { at CDARS Outcome } \\
\text { ADHD after at least } 6 \\
\text { years follow-up (ICD- } \\
\text { 9-CM code 314) until } \\
\text { December 2015 }\end{array}$ & $\begin{array}{c}\text { 5,659 children (3\%) had } \\
\text { a diagnosis of ADHD. } \\
\text { Adjusted HR, ADHD, any } \\
\text { AD users in pregnancy } \\
\text { vs. non-users: } 1.39 \text { (1.07- } \\
\text { 1.82); 1st trimester } 1.43 \\
\text { (1.05-1.95), 2nd trimester } \\
\text { 1.50 (1.08-2.09), 3rd } \\
\text { trimester 1.43 (1.03- } \\
\text { 1.98). Prenatal SSRI 1.11 } \\
\text { (0.77 to 1.60), prenatal } \\
\text { non-SSRI 0.59 (1.19-2.14). } \\
\text { 1st trimester 1.64 (1.13- } \\
\text { 2.38), 2nd trimester 1.72 } \\
\text { (1.16-2.53), 3rd trimester } \\
\text { 1.65 (1.13-2.40). Sibling } \\
\text { analysis: prenatal AD } \\
\text { 0.54 (0.17-1.74); AD non- } \\
\text { users: maternal mental } \\
\text { disease vs. no disease: } \\
\text { 1.84 (1.54-2.18) }\end{array}$ & $\begin{array}{l}\text { Relatively large } \\
\text { population based } \\
\text { cohort. Prescription/ } \\
\text { dispensing data } \\
\text { may not reflect } \\
\text { actual use. Possible } \\
\text { misclassification bias. } \\
\text { Residual confounding } \\
\text { by indication }\end{array}$ & 8 & $\begin{array}{l}\text { Increased risk of ADHD } \\
\text { in children prenatally } \\
\text { exposed to ADs and non- } \\
\text { SSRIs but not to SSRI. } \\
\text { Associations could be at } \\
\text { least in part explained } \\
\text { by maternal depression } \\
\text { or confounding by } \\
\text { indication of AD }\end{array}$ \\
\hline $\begin{array}{l}\text { Boukhris et al. } 57 \\
\text { (2017, Canada) }\end{array}$ & $\begin{array}{l}\text { SSRI, SNRI, MAOI, } \\
\text { TCA (1 st \& 2nd/3rd } \\
\text { trimesters) }\end{array}$ & $\begin{array}{l}\text { Population-based } \\
\text { cohort study with data } \\
\text { from ongoing Quebec } \\
\text { Pregnancy Children } \\
\text { Cohort. All full-term } \\
\text { singletons born alive (n } \\
\text { = 144,406) in Quebec- } \\
\text { Canada in January } \\
\text { 1998-December 2009. } \\
\text { Exposure: mothers } \\
\text { with at least one AD } \\
\text { prescription during } \\
\text { pregnancy recorded in } \\
\text { the Prescription Drug } \\
\text { database. Outcome: } \\
\text { ADHD diagnosis. } \\
\text { Follow-up: birth up to } \\
\text { first diagnosis, death } \\
\text { or December 31, } 2009 \text {, } \\
\text { whichever occurred first }\end{array}$ & $\begin{array}{c}\text { 4,564 infants (3.2\%) } \\
\text { with a diagnosis of } \\
\text { ADHD. Mean age at } \\
\text { first diagnosis } 6.3 \\
\text { years. Adjusted HR: AD } \\
\text { 1st trimester } 1.0 \text { (0.9- } \\
\text { 1.2); 2nd/3rd trimesters } \\
\text { 1.3 (1.0-1.6). 2nd/3rd } \\
\text { trimesters: SSRI 1.2 (0.9- } \\
\text { 1.6), SNRI 1.4 (0.8-2.5), } \\
\text { TCA 1.8 (1.0-3.1), other } \\
\text { ADs 0.5 (0.1-2.2) }\end{array}$ & $\begin{array}{l}\text { Large population } \\
\text { based cohort study. } \\
\text { Prescription filling } \\
\text { data may not reflect } \\
\text { actual use. Lack } \\
\text { of information on } \\
\text { several potential } \\
\text { confounders } \\
\text { (maternal lifestyle, } \\
\text { smoking). Residual } \\
\text { confounding by } \\
\text { indication remained }\end{array}$ & 8 & $\begin{array}{l}\text { After taking into account } \\
\text { maternal history of } \\
\text { depression/anxiety, } \\
\text { prenatal exposure to } \\
\text { AD, especially in the } \\
\text { 2nd/3rd trimesters, } \\
\text { was associated with } \\
\text { increased risk of ADHD }\end{array}$ \\
\hline
\end{tabular}

95\%Cl: 95\% confidence interval; ADHD: attention deficit/hyperactivity disorder; ADs: antidepressants; ASD: autism spectrum disorders; BUP: bupropion; EHR: electronic health record; HR: hazard ratio; ICD: International Classification of Diseases; ICD-CM: International Classification of Diseases - Clinical Modification; MAOI: monoamine oxidase inhibitor; NOS: Newcastle-Ottawa Scale; OR: odds ratio; SNRI: serotonin-norepinephrine reuptake inhibitors; SSRI: selective serotonin reuptake inhibitors; TCA: tricyclic antidepressants. 
Table 3

Maternal use of antidepressant drugs in pregnancy and developmental and cognitive deficits in infant and toddlers.

\begin{tabular}{|c|c|c|c|c|}
\hline Study (year, country) & Drug & $\begin{array}{l}\text { Pregnancy } \\
\text { exposure }\end{array}$ & $\begin{array}{c}\text { Age at } \\
\text { evaluation }\end{array}$ & Results and conclusions/remarks \\
\hline $\begin{array}{l}\text { Nulman et al. } 61 \\
\text { (1997, Canada) }\end{array}$ & TCA, FLX & $\begin{array}{l}1 \text { st, } 2 \text { nd } \& \text { 3rd } \\
\text { trimesters }\end{array}$ & $\begin{array}{l}16 \text { to } 86 \\
\text { months }\end{array}$ & $\begin{array}{c}\text { TCA and FLX in pregnancy did not affect global IQ, language development or } \\
\text { behavioral development in preschool children }\end{array}$ \\
\hline $\begin{array}{l}\text { Nulman et al. } 62 \\
(2002, \text { Canada) }\end{array}$ & TCA, FLX & $\begin{array}{l}\text { 1st, 2nd \& 3rd } \\
\text { trimesters }\end{array}$ & $\begin{array}{l}15 \text { to } 71 \\
\text { months }\end{array}$ & $\begin{array}{c}\text { TCA and FLX in pregnancy did not affect child's global IQ, language } \\
\text { development, or behavior development. IQ was negatively associated with } \\
\text { duration of maternal depression and language with number of depressive } \\
\text { episodes after delivery }\end{array}$ \\
\hline $\begin{array}{l}\text { Reebye et al. } 71 \\
\text { (2002, Canada) }\end{array}$ & $\begin{array}{l}\mathrm{SSRI} / \\
\mathrm{SSRI}+\mathrm{CZP}\end{array}$ & Pregnancy & 3 months & $\begin{array}{l}\text { Exposure to SSRI and SSRI+CZP in pregnancy did not alter scores for infant } \\
\text { development at the age of } 2 \text { months as evaluated by the BSID }\end{array}$ \\
\hline $\begin{array}{l}\text { Casper et al. } 64 \\
(2003, \text { USA) }\end{array}$ & SSRI & Pregnancy & $\begin{array}{l}\text { At birth and } 6 \\
\text { to } 40 \text { months }\end{array}$ & $\begin{array}{l}\text { SSRI exposed newborns showed lower } 1 \text { and } 5 \text { min Apgar scores. SSRI } \\
\text { infants had slightly delayed psychomotor development (adjusted for Apgar). } \\
\text { No difference in mental development. SSRI in breast milk not ruled out }\end{array}$ \\
\hline $\begin{array}{l}\text { Misri et al. } 80 \\
\text { (2006, Canada) }\end{array}$ & $\begin{array}{c}\text { SSRI/ } \\
\text { SSRI+CZP }\end{array}$ & Pregnancy & $\begin{array}{l}48 \text { to } 60 \\
\text { months }\end{array}$ & $\begin{array}{l}\text { Levels of internalizing behaviors (fearfulness, social withdrawal and somatic } \\
\text { complaints) did not differ between SSRI and SSRI+CZP and non-exposed } \\
\text { at age of } 4 \text { years. Increased maternal anxiety/depression associated with } \\
\text { increased internalizing behaviors in their children }\end{array}$ \\
\hline $\begin{array}{l}\text { Oberlander et al. } 79 \\
\text { (2007, Canada) }\end{array}$ & SSRI & Pregnancy & 48 months & $\begin{array}{l}\text { Externalizing (physical/verbal aggressiveness, disruptive behavior) and } \\
\text { attentional behaviors did not differ between prenatal SSRI exposed and } \\
\text { non-exposed 4-year-old children. Current maternal mood/stress predicts } \\
\text { child externalizing behavior regardless prenatal SSRI and depression }\end{array}$ \\
\hline $\begin{array}{l}\text { Casper et al. } 72 \\
(2011, \text { USA) }\end{array}$ & SSRI & $\begin{array}{l}\text { 1st, 2nd \& 3rd } \\
\text { trimesters }\end{array}$ & $\begin{array}{l}\text { At birth and } 14 \\
\text { months }\end{array}$ & $\begin{array}{c}\text { SSRI associated with lower Apgar. SSRI increased risks for lower } \\
\text { psychomotor test scores. Mental development and motor function by } \\
\text { neurological examination within the normal range }\end{array}$ \\
\hline $\begin{array}{l}\text { Salisbury et al. } 98 \\
(2011, \text { USA) }\end{array}$ & SSRI & Pregnancy & $\begin{array}{l}\text { At birth and } 3 \\
\text { weeks }\end{array}$ & $\begin{array}{l}\text { SSRI associated with lower GA at birth. Controlling for GA, SSRI exposed had } \\
\text { lower quality of movements and more CNS stress signs }\end{array}$ \\
\hline $\begin{array}{l}\text { Nulman et al. } 63 \\
(2012 \text {, Canada) }\end{array}$ & $\begin{array}{l}\text { SNRI (VLX), } \\
\text { SSRI }\end{array}$ & $\begin{array}{l}1 \mathrm{st}, 2 \text { nd \& } 3 r d \\
\text { trimesters }\end{array}$ & $\begin{array}{l}36 \text { to } 83 \\
\text { months }\end{array}$ & $\begin{array}{c}\text { Offspring from VLX, SSRI and untreated maternal depression groups had } \\
\text { similar full-scale IQs. VLX and SSRI had lower IQs than children born to non- } \\
\text { depressed women }\end{array}$ \\
\hline $\begin{array}{l}\text { Weikum et al. } 99 \\
\text { (2012, Canada) }\end{array}$ & $\begin{array}{l}\text { SNRI (VLX), } \\
\text { SSRI }\end{array}$ & Pregnancy & $\begin{array}{l}\text { Still in utero, } \\
6 \text { and } 10 \\
\text { months }\end{array}$ & $\begin{array}{l}\text { As expected, children from non-depressed non-treated mothers succeed } \\
\text { the non-native speech and visual language discrimination test at } 6 \text { and } \\
\text { failed it at } 10 \text { months whereas SSRI exposed succeeded it at both ages. } \\
\text { As expected, control fetuses responded to vowel but not to consonant } \\
\text { discrimination, whereas SSRI-exposed responded for both vowel and } \\
\text { consonant }\end{array}$ \\
\hline $\begin{array}{l}\text { Austin et al. } 65 \\
\text { (2013, Australia) }\end{array}$ & $\begin{array}{l}\text { ADs/mostly } \\
\text { SSRI }\end{array}$ & $\begin{array}{l}\text { 1st, 2nd \& 3rd } \\
\text { trimesters }\end{array}$ & $17-24$ months & $\begin{array}{l}\text { ADs (mostly SSRI) not associated (BSID-III) with poorer cognitive, language or } \\
\text { motor development outcomes in 18-month-old infants }\end{array}$ \\
\hline $\begin{array}{l}\text { Batton et al. } 73 \\
\text { (2013, Canada) }\end{array}$ & SSRI & Pregnancy & 24-36 months & $\begin{array}{c}\text { Cohort of preterm born infants: antenatal SSRI did not increase risk of } \\
\text { adverse neurodevelopment (BSID) above the baseline risk for this degree of } \\
\text { prematurity }\end{array}$ \\
\hline $\begin{array}{l}\text { Hanley et al. } 100 \\
(2013, \text { Canada) }\end{array}$ & SRI & Pregnancy & 10 months & $\begin{array}{l}\text { SRI-exposed 10-month-old infants scored lower than non-exposed on gross- } \\
\text { motor, social-emotional and adaptive behaviors (BSID). Controlled for pre/ } \\
\text { postnatal maternal depression }\end{array}$ \\
\hline $\begin{array}{l}\text { Pedersen et al. } 76 \\
\text { (2013, Denmark) }\end{array}$ & ADs & $\begin{array}{l}\text { 1st, 2nd \& 3rd } \\
\text { trimesters }\end{array}$ & $\begin{array}{l}48 \text { to } 60 \\
\text { months }\end{array}$ & $\begin{array}{l}\text { Children behavior assessed by parent reported SDQ. AD not associated with } \\
\text { abnormal SDQ scores. Prenatal untreated depression associated abnormal } \\
\text { SDQ }\end{array}$ \\
\hline $\begin{array}{l}\text { de Vries et al. } 101 \\
(2013, \text { Netherlands) }\end{array}$ & SSRI & Pregnancy & $\begin{array}{c}2 \text { and } 7 \text { days, } 3 \\
\text { to } 4 \text { months }\end{array}$ & $\begin{array}{l}\text { Prenatal SSRI impaired quality of infant movements (assessed by Prechtl } \\
\text { and motor optimality scales) irrespective of maternal depression/anxiety. } \\
\text { Severity of maternal depression remained as residual confounding }\end{array}$ \\
\hline $\begin{array}{l}\text { Santucci et al. } 74 \\
(2014, \text { USA) }\end{array}$ & $\mathrm{SRI}$ & Pregnancy & $\begin{array}{c}12,26,52 \text { and } \\
78 \text { weeks }\end{array}$ & $\begin{array}{c}\text { No impact of prenatal SRI or maternal depression on mental (cognitive) } \\
\text { development index scores. Effect of SRI on (first year) psychomotor } \\
\text { development scores, a possibly transitory effect }\end{array}$ \\
\hline
\end{tabular}

(continues) 
Table 3 (continued)

\begin{tabular}{|c|c|c|c|c|}
\hline Study (year, country) & Drug & $\begin{array}{l}\text { Pregnancy } \\
\text { exposure }\end{array}$ & $\begin{array}{c}\text { Age at } \\
\text { evaluation }\end{array}$ & Results and conclusions/remarks \\
\hline $\begin{array}{l}\text { Skurtveit et al. } 75 \\
(2014, \text { Norway) }\end{array}$ & SSRI & $\begin{array}{l}\text { Pregnancy up } \\
\text { to } 6 \text { month } \\
\text { old }\end{array}$ & 36 months & $\begin{array}{l}\text { SSRI in pregnancy associated with lower language competence (measured } \\
\text { by mother's report on language-grammar scale) at } 3 \text { years independently } \\
\text { of anxiety/depression before/during pregnancy. Anxiety/depression in } \\
\text { pregnancy also associated with language competence delay }\end{array}$ \\
\hline $\begin{array}{l}\text { Eriksen et al. } 102 \\
(2015, \text { Denmark) }\end{array}$ & $\begin{array}{l}\text { ADs/ } \\
\text { anxiolytics }\end{array}$ & Pregnancy & $60-64$ months & $\begin{array}{l}\text { No association between children's IQ (Wechsler Preschool and Primary Scale } \\
\text { of Intelligence) and prenatal exposure to ADs and anxiolytics. Low statistical } \\
\text { power study }\end{array}$ \\
\hline $\begin{array}{l}\text { Hanley, et al. } 78 \\
(2015, \text { Canada) }\end{array}$ & SRI & Pregnancy & 36-72 months & $\begin{array}{l}\text { SRI in pregnancy was associated with increased internalizing and } \\
\text { anxious behavior (after controlling for maternal depression) but not with } \\
\text { externalizing behavior in early childhood }\end{array}$ \\
\hline $\begin{array}{l}\text { Brown et al. } 103 \\
(2016, \text { Finland })\end{array}$ & SSRI & $\begin{array}{l}30 \text { days } \\
\text { before, } 1 \text { st, } \\
2^{\text {nd }} \& 3 \text { rd } \\
\text { trimesters }\end{array}$ & $\begin{array}{l}<14 \text { year old, } \\
\text { most }<9 \text { years }\end{array}$ & $\begin{array}{l}\text { Children born to mothers who used SSRI during pregnancy had an } \\
\text { increased risk of speech/language disorders compared with the offspring of } \\
\text { mothers diagnosed as having depression or other psychiatric disorders not } \\
\text { treated with ADs }\end{array}$ \\
\hline $\begin{array}{l}\text { Grzeskowiak et al. } 77 \\
(2016, \text { Denmark) }\end{array}$ & $\begin{array}{l}\text { SSRI, SNRI, } \\
\quad \text { TCA }\end{array}$ & $\begin{array}{l}1 \text { st, 2nd \& 3rd } \\
\text { trimesters }\end{array}$ & $\begin{array}{l}48,60,84 \\
\text { months }\end{array}$ & $\begin{array}{l}\text { SSRI in pregnancy did not increase risks of behavioral problems (SDQ) } \\
\text { in 7-year-old children after adjustment for maternal illness. Untreated } \\
\text { prenatal depression increased risks of behavioral problems compared with } \\
\text { unexposed children }\end{array}$ \\
\hline $\begin{array}{l}\text { Handal et al. } 104 \\
(2016, \text { Norway) }\end{array}$ & SSRI & $\begin{array}{l}180 \text { days } \\
\text { before and } \\
\text { pregnancy }\end{array}$ & 36 months & $\begin{array}{l}\text { SSRIs during pregnancy was weakly associated with a delayed motor } \\
\text { development (Ages \& Stages Questionnaire) at age of } 3 \text { years, but the delay } \\
\text { was very short and of no clinical importance }\end{array}$ \\
\hline $\begin{array}{l}\text { Handal et al. } 70 \\
(2016, \text { Norway) }\end{array}$ & SSRI & Pregnancy & 36 months & $\begin{array}{l}\text { SSRIs plus folic acid supplementation in pregnancy increased risks of } \\
\text { delayed language competence in the offspring. SSRI without folic acid did } \\
\text { not alter risks of delayed language competence. Unexpected interaction }\end{array}$ \\
\hline $\begin{array}{l}\text { Johnson et al. } 66 \\
(2016, \text { USA) }\end{array}$ & SRI & Pregnancy & $30-66$ months & $\begin{array}{l}\text { Prenatal SRI weakly associated with some preschool outcomes (expressive } \\
\text { language and behavior problems) but not with cognitive function }\end{array}$ \\
\hline $\begin{array}{l}\text { Hermansen et al. } 67 \\
(2016, \text { Norway) }\end{array}$ & SSRI & Pregnancy & $60-72$ months & $\begin{array}{l}\text { No effects of prenatal depression or SSRIs upon general cognition or } \\
\text { inhibition. Both SSRI and maternal depression associated with higher levels } \\
\text { of externalizing behaviors compared to non-exposed controls. SSRI exposed } \\
\text { children showed higher levels of internalizing behaviors }\end{array}$ \\
\hline $\begin{array}{l}\text { Salisbury et al. } 105 \\
(2016, \text { USA) }\end{array}$ & $\begin{array}{c}\mathrm{SSRI} / \\
\mathrm{SSRI}+\mathrm{BZP}\end{array}$ & Pregnancy & $\begin{array}{l}\text { 1st postnatal } \\
\text { month }\end{array}$ & $\begin{array}{l}\text { Infants of pregnant mothers treated with SSRI and SSRI+BZP had lower } \\
\text { motor scores and more CNS stress signs. Infants of depressed mothers } \\
\text { had low arousal throughout the 30-day period. Infants of SSRI+BZP group } \\
\text { had the least favorable scores on the Neonatal Intensive Care Unit Network } \\
\text { Neurobehavioral Scale }\end{array}$ \\
\hline $\begin{array}{l}\text { Hermansen, et al. } 106 \\
(2017, \text { Norway) }\end{array}$ & SSRI & Pregnancy & $\begin{array}{l}\text { Circa } 68 \\
\text { months }\end{array}$ & $\begin{array}{l}\text { Prenatal exposure to SSRIs and depression was not directly associated with } \\
\text { abilities of interference suppression (behavioral flanker task while recording } \\
\text { event-related potential ERPs) }\end{array}$ \\
\hline $\begin{array}{l}\text { El Marroun et al. } 68 \\
(2017, \text { Netherlands) }\end{array}$ & SSRI & Pregnancy & $\begin{array}{l}48,60 \text { and } 84 \\
\text { months }\end{array}$ & $\begin{array}{l}\text { SSRI in pregnancy was not related to maternal reported executive function } \\
\text { at } 4 \text { years, nor was it related with observed non-verbal intelligence at age } \\
5 \text { or neuropsychological function at } 7 \text { years. SSRI and untreated maternal } \\
\text { depression in pregnancy had no major impact on child non-verbal cognition }\end{array}$ \\
\hline $\begin{array}{l}\text { Viktorin et al. } 69 \\
\text { (2017, Sweden) }\end{array}$ & ADs/SSRI & Pregnancy & $\begin{array}{l}\text { Circa } 96 \\
\text { months }\end{array}$ & $\begin{array}{l}\text { No evidence of association between maternal AD use in pregnancy and } \\
\text { child intellectual disability after adjustment for confounding factors }\end{array}$ \\
\hline $\begin{array}{l}\text { Lupatelli et al. } 81 \\
\text { (2018, Norway) }\end{array}$ & ADs/SSRI & Pregnancy & $\begin{array}{l}18 \text { to } 60 \\
\text { months }\end{array}$ & $\begin{array}{l}\text { SSRI in pregnancy did not increase risks for externalizing, emotional, or } \\
\text { social problems in preschool-aged children }\end{array}$ \\
\hline
\end{tabular}

AD: antidepressant drug; BSID: Bayley Scales of Infant Development; BZP: benzodiazepine; CNS: central nervous system; CZP: clonazepam; ERP: event-related potentials; FLX: fluoxetine; GA: gestational age; IQ: intelligence quotient; SDQ: Strengths and Difficulties Questionnaire; SNRI: serotonin norepinephrine reuptake inhibitor; SRI: serotonin reuptake inhibitor; SSRI: selective serotonin reuptake inhibitors; TCA: tricyclic antidepressant; VLX: venlafaxine. 
treated with ADs or not during pregnancy, may slightly increase the risks of deficits of IQ and language development 62 , language competence 75 , and/or behavior development 76,77 .

Six studies evaluated the impact of prenatal ADs (mostly SSRI) on levels of internalizing and/or externalizing behaviors in preschool children. One study found that both prenatal SSRI and depression increased risks of externalizing behaviors while SSRI increased risks of internalizing behaviors 67 . Another investigation indicated that prenatal SRI predicts internalizing but not externalizing behaviors in early childhood 78 . Oberlander et al. 79 found that antenatal SSRI and depression are not associated with the child's externalizing behavior while current maternal mood predicts it. Two additional studies found no association between prenatal SSRIs or ADs with internalizing 80 and externalizing behaviors 81 . Overall, the foregoing findings showed no consistent association of antenatal SSRIs and depression with children's internalizing or externalizing behaviors.

\section{Discussion}

\section{Residual confounding by indication and severity of depression}

A residual confounding by indication was a shortcoming with major impact on the interpretation of most research findings. ADs are indicated mainly for the treatment of depressive disorders and so the possibility exists that associations resulted from the maternal depression for which the drug was prescribed and not from the pharmacological intervention. In other words, reported associations between children's neurodevelopmental disorders (ASD and ADHD) and maternal use of ADs in pregnancy may be non-causal. A few studies did not control confounding by indication at all and simply compared the offspring of AD-treated depressed mothers with the offspring of untreated and non-depressed pregnant women. These studies generally found a higher risk of ASD/ADHD among children prenatally exposed to ADs. In most studies, however, investigators made an attempt to control this confounding in the statistical analysis by adjusting risk ratios for effects of dichotomous presence or absence of maternal depression or history of psychiatric disorders, or yet, by also making a maternal sibling sub-cohort analysis.

It is fair to think that depression entailing prescription for $\mathrm{AD}$ is probably more severe than the disorder of patients not receiving pharmacological intervention. The lack of adjustment for severity of depression may have led to spurious associations of AD exposure in pregnancy with any analyzed outcome, if there is a causal link between the outcome (ASD, ADHD or other neurodevelopmental disorder) and the mother's major depressive illness.

\section{Depression and risks of ASD, ADHD and neurodevelopmental impairment}

The notion that maternal depression might be an independent risk factor for adverse pregnancy outcomes and offspring neurodevelopmental disorders is not only plausible but it is also consistent with findings from observational studies. Systematic reviews, with or without meta-analysis, found that untreated depression, anxiety and/or perceived stress during pregnancy were associated with small increases in the risk of adverse pregnancy outcomes such as preterm births, low birth weights and small-for-gestational-age infants 82,83 . Untreated depression also causes effects on the developing fetus such as hyperactivity, irregular fetal heart rate and altered EEG patterns 84 . The impact of maternal depression on child development and mental health has been far less explored. Nonetheless, some researches provided indirect evidence for such association of unmedicated maternal depression with ASD. A strong evidence along this line was provided by studies showing that preconceptiononly maternal exposure to ADs increased risks of ASD in the offspring 36,37,41,45. AD therapy before pregnancy indicates that these women suffered from a depressive disorder severe enough to require a pharmacological intervention. It is difficult to foresee, on the other hand, how a preconception-only exposure to ADs could have any detrimental effect on the further development of the conceptus. Moreover, associations of ASD with AD use in pregnancy detected by several studies proved to be nonsignificant after adjustment for maternal psychiatric disorders. Recently, Hagberg et al. 43 found that untreated depression, but not $\mathrm{AD}$ use in pregnancy for disorders other than depression, increased 
risks of ASD in the offspring. Using the duration of the last episode of depression as a proxy for disorder severity, the authors noted that risk of ASD increased with increasing severity of maternal depression. Collectively, these findings are consistent with the interpretation that a maternal history of depression might be an independent risk factor for ASD.

Seven studies found that maternal depression in pregnancy and/or after birth, regardless of whether it is treated or untreated, might impair child development 62,67,75,76,77,79,80. These findings showed that, as noted for ASD and ADHD, the non-adjustment of risk ratios for severity of depression may have led to spurious associations of antenatal AD with deficits in the cognitive and behavioral development of infants and preschool children.

\section{Genetics and mother-child interaction}

Both inheritable genetic traits and depression-caused abnormal mother-child interaction might provide plausible explanations for associations between maternal depression and enhanced risks of neurodevelopmental and psychiatric disorders in the offspring.

Affective disorders have well-established genetic components and family studies indicated 2to 3-fold increases in lifetime risk of major depression among first-degree relatives 85,86 . A recent genome-wide association meta-analysis identified 44 genetic risk loci for major depressive disorders and strongly suggested the existence of biological processes common to major depression and schizophrenia. The genome analysis indicated that some biological processes might be common to major depression and other psychiatric disorders such as ASD and ADHD ${ }^{86}$. In the study by Malm et al. 35, for instance, inherited risk factors for major depression were lurking variables that might have influenced the apparent association between SSRI use in pregnancy and depressive disorders in the adolescent offspring. Although the association was significant after adjustment for maternal psychiatric illness, the authors did not adjust risk ratios for severity of maternal depression. SSRI use in pregnancy could have been a surrogate or marker for more severe maternal depressive disorders and, as mentioned, major depression has a genetic and inheritable component.

Furthermore, various studies provided evidence that depression can adversely affect mother-child bonding as well as the child's development and mental health 87 .

Sibling analysis designs are useful to investigate associations thought likely to suffer confounding arising from genetics and environmental postnatal factors, including the detrimental effects of maternal depressive symptoms on mother-child interaction. In principle, such strategy to control potential confounding in the design of the study seems to be better than merely adjusting risk ratios in the statistical analysis using dichotomous data on the presence/absence of maternal history of depression. Nonetheless, a possible disadvantage of sibling analysis in a sub-cohort of population-based cohorts is that it drastically reduces the sample size and thus the statistical power of the analysis. In all sibling analyses conducted by reviewed studies, no association of ADs in pregnancy with ASD was found 38,40,42,43, nor was it detected with ADHD 42,51,58.

\section{Non-differential misclassification of exposure}

A notable shortcoming common to all reviewed studies is a potential non-differential misclassification of exposure status due to lack of confirmation of the actual drug intake in pregnancy. Exposure assessment (binary exposure) took into account records of drug prescription and dispensing, but no study confirmed patients' adherence to AD treatment. The exposure status relevant for the outcome of interest is not merely a matter of using or not ADs during pregnancy. The effect of exposure on the outcome is likely to depend on the magnitude (dose), timing and adherence to prescribed pharmacotherapy. Nonadherence to prescribed medication and, particularly, poor AD adherence is a challenging issue in psychiatric practice. It is estimated that up to approximately $50 \%$ of psychiatric patients, including those with major depression prematurely discontinue drug therapy 88,89 . Nonadherence with medication is also a common problem among pregnant women whose drug-taking behavior can be negatively influenced by concerns about harmful effects of the pharmacological therapy on the unborn child, among other factors ${ }^{90}$. The influence of concomitant pregnancy and depressive illness on the high rates of nonadherence with medication noted in either 
condition alone is unclear. At any rate, non-differential misclassification of exposure is likely to bias towards the null estimates of detrimental effects of prenatal AD exposure on offspring's health.

\section{National scenarios}

Another potential drawback of the reviewed cohort or case-control studies is the fact that nearly all of them used data from nationwide (or territory-based) health registries (e.g., Scandinavian countries, Finland, Hong Kong), or large databases from health insurance companies (e.g., United States, United Kingdom). These studies investigated associations between exposure and outcomes in people living in a few highly developed countries and findings might be somewhat different for distinct exposure scenarios and populations from Asia, Africa and Latin America.

\section{Publication bias and searching strategy}

In principle, publication biases may misdirect the qualitative and/or quantitative synthesis of any systematic review. It is unlikely, however, that any good quality observational study on the topic addressed by this systematic review would have remained unpublished. The possibility exists, on the other hand, that the adopted searching strategy (search strings and databases) was not effective to identify all relevant studies. To verify whether the search strategy failed to identify articles of interest, we compared the set of studies included in this review with those analyzed by previous systematic reviews. Nine reviews conducted between 2014 and 2018 examined a possible association of ADs in pregnancy with ASD 16,17,18,19,20,21,22,23,24. Our review about ASD risks covered not only all studies included in the previous reviews but also analyzed two more studies than the most recently published review. Two reviews, both published in 2018, addressed the risks of ADHD 26,27. Again, this study covered not only all studies included in published reviews but one more than those examined by one of the two previous reviews. Two reviews published in 201191 and 201828 , including 5 and 7 studies, respectively, evaluated risks of neurodevelopmental disorders arising from prenatal exposures to ADs. Except for one study 92, this review analyzed all studies included in previous reviews and 23 additional articles. Suri et al. 92 found no association of AD in pregnancy with neurobehavioral outcomes and their study was included in the review by Prady et al. 28 .

The foregoing comparison with previous reviews suggests that this updated review, encompassing the three outcomes of interest, did not fail to retrieve and include any relevant study about potential associations between prenatal exposure to AD and risks of ASD, ADHD and neurodevelopmental disorders.

\section{SSRIs and neurodevelopmental outcomes}

Studies of associations of ADs in pregnancy with impaired neurocognitive and behavioral development during infancy and early childhood were heterogeneous regarding the design, instruments used to assess neurocognitive development outcomes and child age at assessment. Overall, their findings indicated that antenatal exposure to SSRI did not impair further child development. Many of these studies used scales to assess child development such as the Bayley Scales of Infant Development (BSID) for infants with ages ranging from one to 42 months. BSID has high reliability and validity but, unless the scores are very low, its predictive value for long-term intellectual and motor disabilities is questionable $93,94,95$. The clinical relevance of significant yet small differences in BDSI scores detected by some studies is unclear.

Since observational studies have limitations for controlling confounding and making causal inferences, a prospective, randomized, placebo-controlled, double-blinded clinical trial is under way in Sweden to clarify whether a SSRI (sertraline) and/or depression in pregnancy might in fact affect child neurocognitive development. The primary objective of this trial is to assess cognitive development at 2 years of age using the BSID-III scale. The recruitment of pregnant women for this study (MAGDALENA study protocol) will be finished in 5 to 6 years' time 96 . 


\section{Concluding remarks}

This study found no consistent evidence of association between antenatal exposure to ADs and increased risks of ASD, ADHD, psychiatric illnesses, and cognitive and or developmental deficits in preschool children.

The conclusion that prenatal exposure to SSRIs and/or serotonin-norepinephrine reuptake inhibitors (SNRIs) does not predict risks of ASD is particularly robust. It is of note that results from some studies strongly suggested that maternal depression, regardless of whether it is treated or untreated during pregnancy, increases risks of ASD in the offspring. A recent population-based cohort study (mother-newborn pairs from Manitoba, Canada, born 1996-2009 with follow-up through 2014) found no association ( $\mathrm{HR}=0.92$; 95\%CI: 0.42-2.03) between antenatal exposure to SSRI or SNRI and ASD 97. This study was not included because only a conference abstract was available when the review was completed.

As commented before, eight reviews of observational studies found a positive association of ASD with use of SSRIs in pregnancy 16,17,18,19,20,21,22,23. At least four of these studies highlighted that maternal psychiatric condition was a major confounding 18,20,22,23 or that causality remained to be confirmed 17 . Two additional reviews found no association 24 , or pointed out that a residual confounding by indication and inconsistent findings precluded a conclusion about risks of ASD 25. Along the same line, three reviews concluded that a residual confounding by indication cannot be ruled out as an explanation for observed associations of prenatal AD exposure with ADHD 25,26,27. Only one review 28 evaluated studies that compared behavioral and neurodevelopmental outcomes for children whose mothers took ADs during pregnancy with those whose mothers suffered from mental disorders but did not take medication. This study 28 found very limited evidence indicating that gestational use of ADs might impair behavioral and neurodevelopmental outcomes in the offspring.

Overall, findings from this study and those from other systematic reviews addressing risks of poor pregnancy outcomes and developmental disorders do not support concerns on the risks of SSRIs/ SNRIs for the unborn child.

\section{Contributors}

J. S. A. Araujo contributed to the conception and study design, data collection and analysis, and drafting of the manuscript. I. F. Delgado contributed to the study design, data analysis, and review of manuscript. F. J. R. Paumgartten conceived and designed the study, contributed for data collection and interpretation and wrote a first draft of the manuscript. The final version of the article submitted for publication was approved by all authors.

\section{Additional informations}

ORCID: Jessica Salvador Areias de Araujo (00000003-0944-1067); Isabella Fernandes Delgado (0000-0003-0610-5324); Francisco José Roma Paumgartten (0000-0002-6207-0149).

\section{References}

1. Richards D. Prevalence and clinical course of depression: a review. Clin Psychol Rev 2011; 31:1117-25.

2. World Health Organization. Depression and other common mental disorders. Global health estimates. Geneva: World Health Organization; 2017.

3. Biaggi A, Conroy S, Pawlby S, Pariante CM. Identifying the women at risk of antenatal anxiety and depression: a systematic review. J Affect Disord 2016; 191:62-77.

4. Vigod SN, Wilson CA, Howard LM. Depression in pregnancy. BMJ 2016; 352:i1547.

5. Byatt N, Xiao RS, Dinh KH, Waring ME. Mental health care use in relation to depressive symptoms among pregnant women in the USA. Arch Womens Ment Health 2016; 19:187-91.

6. Campagne DM. Antidepressant use in pregnancy: are we closer to consensus? Arch Womens Ment Health 2019; 22:189-97. 
7. Gao SY, Wu QJ, Sun C, Zhang TN, Shen ZQ, Liu CX, et al. Selective serotonin reuptake inhibitor use during early pregnancy and congenital malformations: a systematic review and meta-analysis of cohort studies of more than 9 million births. BMC Med 2018; 16:205.

8. Källén B. Neonate characteristics after maternal use of antidepressants in late pregnancy. Arch Pediatr Adolesc Med 2004; 158:312-6.

9. Gentile S. Tricyclic antidepressants in pregnancy and puerperium. Expert Opin Drug Saf 2014; 13:207-25.

10. Mitchell J, Goodman J. Comparative effects of antidepressant medications and untreated major depression on pregnancy outcomes: a systematic review. Arch Womens Ment Health 2018; 21:505-16.

11. Rotem-Kohavi N, Oberlander TF. Variations in neurodevelopmental outcomes in children with prenatal SSRI antidepressant exposure. Birth Defects Res 2017; 109:909-23.

12. Rice D, Barone Jr. S. Critical periods of vulnerability for the developing nervous system: evidence from humans and animal models. Environ Health Perspect 2000; 108 Suppl 3:511-33.

13. Lesch KP, Waider J. Serotonin in the modulation of neural plasticity and networks: implications for neurodevelopmental disorders. Neuron 2012; 76:175-91.

14. Rotem-Kohavi N, Williams LJ, Virji-Babul N, Bjornson BH, Brain U, Werker JF, et al. Alterations in resting-state networks following in utero selective serotonin reuptake inhibitor exposure in the neonatal brain. Biol Psychiatry Cogn Neurosci Neuroimaging 2019; 4:39-49.

15. Sujan AC, Öberg AS, Quinn PD, D’Onofrio BM. Annual research review: maternal antidepressant use during pregnancy and offspring neurodevelopmental problems - a critical review and recommendations for future research. J Child Psychol Psychiatry 2019; 60:356-76

16. Rais TB, Rais A. Association between antidepressants use during pregnancy and autistic spectrum disorders: a meta-analysis. Innov Clin Neurosci 2014; 11:18-22.

17. Man KK, Tong HH, Wong LY, Chan EW, Simonoff E, Wong IC. Exposure to selective serotonin reuptake inhibitors during pregnancy and risk of autism spectrum disorder in children: a systematic review and meta-analysis of observational studies. Neurosci Biobehav Rev 2015; 49:82-9.

18. Kobayashi T, Matsuyama T, Takeuchi M, Ito S. Autism spectrum disorder and prenatal exposure to selective serotonin reuptake inhibitors: a systematic review and meta-analysis. Reprod Toxicol 2016; 65:170-8.

19. Healy D, Le Noury J, Mangin D. Links between serotonin reuptake inhibition during pregnancy and neurodevelopmental delay/ spectrum disorders: a systematic review of epidemiological and physiological evidence. Int J Risk Saf Med 2016; 28:125-41.
20. Kaplan YC, Keskin-Arslan E, Acar S, Sozmen $K$. Prenatal selective serotonin reuptake inhibitor use and the risk of autism spectrum disorder in children: a systematic review and meta-analysis. Reprod Toxicol 2016; 66:31-43.

21. Andalib S, Emamhadi MR, YousefzadehChabok S, Shakouri SK, Høilund-Carlsen PF, Vafaee MS, et al. Maternal SSRI exposure increases the risk of autistic offspring: a metaanalysis and systematic review. Eur Psychiatry 2017; 45:161-6.

22. Mezzacappa A, Lasica PA, Gianfagna F, Cazas O, Hardy P, Falissard B, et al. Risk for autism spectrum disorders according to period of prenatal antidepressant exposure: a systematic review and meta-analysis. JAMA Pediatr 2017; 171:555-63.

23. Kaplan YC, Keskin-Arslan E, Acar S, Sozmen K. Maternal SSRI discontinuation, use, psychiatric disorder and the risk of autism in children: a meta-analysis of cohort studies. Br J Clin Pharmacol 2017; 83:2798-806.

24. Zhou XH, Li YJ, Ou JJ, Li YM. Association between maternal antidepressant use during pregnancy and autism spectrum disorder: an updated meta-analysis. Mol Autism 2018; 9:21.

25. Morales DR, Slattery J, Evans S, Kurz X. Antidepressant use during pregnancy and risk of autism spectrum disorder and attention deficit hyperactivity disorder: systematic review of observational studies and methodological considerations. BMC Med 2018; 16:6.

26. Man KKC, Chan EW, Ip P, Coghill D, Simonoff E, Chan PKL, et al. Prenatal antidepressant exposure and the risk of attention-deficit hyperactivity disorder in children: a systematic review and meta-analysis. Neurosci Biobehav Rev 2018; 86:1-11.

27. Uguz F. Maternal antidepressant use during pregnancy and the risk of attention-deficit/ hyperactivity disorder in children: a systematic review of the current literature. J Clin Psychopharmacol 2018; 38:254-9.

28. Prady SL, Hanlon I, Fraser LK, Mikocka-Walus A. A systematic review of maternal antidepressant use in pregnancy and short- and long-term offspring's outcomes. Arch Womens Ment Health 2018; 21:127-40.

29. Lyall K, Croen L, Daniels J, Fallin MD, LaddAcosta C, Lee BK, et al. The changing epidemiology of autism spectrum disorders. Annu Rev Public Health 2017; 38:81-102.

30. Biederman J, Faraone SV. Attention-deficit hyperactivity disorder. Lancet 2005; 366:237-48.

31. Sayal K, Prasad V, Daley D, Ford T, Coghill D. ADHD in children and young people: prevalence, care pathways, and service provision. Lancet Psychiatry 2018; 5:175-86.

32. Shamseer L, Moher D, Clarke M, Ghersi D, Liberati A, Petticrew M, et al. Preferred reporting items for systematic review and meta-analysis protocols (PRISMA-P) 2015: elaboration and explanation. BMJ 2015; 350:g7647. 
33. von Elm E, Altman DG, Egger M, Pocock SJ, Gøtzsche PC, Vandenbroucke JP, et al. Strengthening the Reporting of Observational Studies in Epidemiology (STROBE) statement: guidelines for reporting observational studies. BMJ 2007; 335:806-8.

34. Wells GA, Shea B, O'Connell D, Peterson J, Welch V, Losos M, et al. The Newcastle-Ottawa Scale (NOS) for assessing the quality of nonrandomized studies in meta-analyses. http://www. ohri.ca/programs/clinical_epidemio logy/oxford.asp (accessed on 11/Dec/2018).

35. Malm H, Brown AS, Gissler M, Gyllenberg D, Hinkka-Yli-Salomäki S, McKeague IW, et al. Gestational exposure to selective serotonin reuptake inhibitors and offspring psychiatric disorders: a national register-based study. J Am Acad Child Adolesc Psychiatry 2016; 55:359-66.

36. Hviid A, Melbye M, Pasternak B. Use of selective serotonin reuptake inhibitors during pregnancy and risk of autism. N Engl J Med 2013; 369:2406-15.

37. Castro VM, Kong SW, Clements CC, Brady R, Kaimal AJ, Doyle AE, et al. Absence of evidence for increase in risk for autism or attention-deficit hyperactivity disorder following antidepressant exposure during pregnancy: a replication study. Transl Psychiatry 2016; 6:e708.

38. Brown HK, Ray JG, Wilton AS, Lunsky Y, Gomes T, Vigod SN. Association between serotonergic antidepressant use during pregnancy and autism spectrum disorder in children. JAMA 2017; 317:1544-52.

39. Viktorin A, Uher R, Reichenberg A, Levine SZ, Sandin S. Autism risk following antidepressant medication during pregnancy. Psychol Med 2017; 47:2787-96.

40. Sørensen MJ, Grønborg TK, Christensen J, Parner ET, Vestergaard M, Schendel D, et al. Antidepressant exposure in pregnancy and risk of autism spectrum disorders. Clin Epidemiol 2013; 5:449-59.

41. Clements CC, Castro VM, Blumenthal SR, Rosenfield HR, Murphy SN, Fava M, et al. Prenatal antidepressant exposure is associated with risk for attention-deficit hyperactivity disorder but not autism spectrum disorder in a large health system. Mol Psychiatry 2015; 20:727-34.

42. Sujan AC, Rickert ME, Öberg AS, Quinn PD, Hernández-Díaz S, Almqvist C, et al. Associations of maternal antidepressant use during the first trimester of pregnancy with preterm birth, small for gestational age, autism spectrum disorder, and attention-deficit/hyperactivity disorder in offspring. JAMA 2017; 317:1553-62.

43. Hagberg KW, Robijn AL, Jick S. Maternal depression and antidepressant use during pregnancy and the risk of autism spectrum disorder in offspring. Clin Epidemiol 2018; 10:1599-612.
44. Croen LA, Grether JK, Yoshida CK, Odouli R, Hendrick V. Antidepressant use during pregnancy and childhood autism spectrum disorders. Arch Gen Psychiatry 2011; 68:1104-12.

45. Gidaya NB, Lee BK, Burstyn I, Yudell M, Mortensen EL, Newschaffer CJ. In utero exposure to selective serotonin reuptake inhibitors and risk for autism spectrum disorder. J Autism Dev Disord 2014; 44:2558-67.

46. Boukhris T, Sheehy O, Mottron L, Bérard A. Antidepressant use during pregnancy and the risk of autism spectrum disorder in children. JAMA Pediatr 2016; 170:117-24.

47. Harrington RA, Lee LC, Crum RM, Zimmerman AW, Hertz-Picciotto I. Prenatal SSRI use and offspring with autism spectrum disorder or developmental delay. Pediatrics 2014; 133:e1241-8.

48. Rai D, Lee BK, Dalman C, Golding J, Lewis G, Magnusson C. Parental depression, maternal antidepressant use during pregnancy, and risk of autism spectrum disorders: population based case-control study. BMJ 2013; 346:f2059.

49. Rai D, Lee BK, Dalman C, Newschaffer C, Lewis G, Magnusson C. Antidepressants during pregnancy and autism in offspring: population based cohort study. BMJ 2017; 358:j2811.

50. El Marroun H, White TJ, van der Knaap NJ, Homberg JR, Fernández G, Schoemaker NK, et al. Prenatal exposure to selective serotonin reuptake inhibitors and social responsiveness symptoms of autism: population-based study of young children. Br J Psychiatry 2014; 205:95-102.

51. Laugesen K, Olsen MS, Telén Andersen AB, Frøslev T, Sørensen HT. In utero exposure to antidepressant drugs and risk of attention deficit hyperactivity disorder: a nationwide Danish cohort study. BMJ Open 2013; 3:e003507.

52. Figueroa R. Use of antidepressants during pregnancy and risk of attention-deficit/hyperactivity disorder in the offspring. J Dev Behav Pediatr 2010; 31:641-8.

53. Dwoskin LP, Rauhut AS, King-Pospisil KA, Bardo MT. Review of the pharmacology and clinical profile of bupropion, an antidepressant and tobacco use cessation agent. CNS Drug Rev 2006; 12:178-207.

54. Zisook S, Rush AJ, Haight BR, Clines DC, Rockett CB. Use of bupropion in combination with serotonin reuptake inhibitors. Biol Psychiatry 2006; 59:203-10.

55. Dunner DL. Combining antidepressants. Shanghai Arch Psychiatry 2014; 26:363-4.

56. Patel K, Allen S, Haque MN, Angelescu I, Baumeister D, Tracy DK. Bupropion: a systematic review and meta-analysis of effectiveness as an antidepressant. Ther Adv Psychopharmacol 2016; 6:99-144.

57. Boukhris T, Sheehy O, Bérard A. Antidepressant use in pregnancy and the risk of attention deficit with or without hyperactivity disorder in children. Paediatr Perinat Epidemiol 2017; 31:363-73. 
58. Man KKC, Chan EW, Ip P, Coghill D, Simonoff E, Chan PKL, et al. Prenatal antidepressant use and risk of attention-deficit/hyperactivity disorder in offspring: population based cohort study. BMJ 2017; 357:j2350.

59. Gogtay N, Vyas NS, Testa R, Wood SJ, Pantelis C. Age of onset of schizophrenia: perspectives from structural neuroimaging studies. Schizophr Bull 2011; 37:504-13.

60. Jones PB. Adult mental health disorders and their age at onset. Br J Psychiatry Suppl 2013; 54:s5-10.

61. Nulman I, Rovet J, Stewart DE, Wolpin J, Gardner HA, Theis JG, et al. Neurodevelopment of children exposed in utero to antidepressant drugs. N Engl J Med 1997; 336:258-62.

62. Nulman I, Rovet J, Stewart DE, Wolpin J, PaceAsciak P, Shuhaiber S, et al. Child development following exposure to tricyclic antidepressants or fluoxetine throughout fetal life: a prospective, controlled study. Am J Psychiatry 2002; 159:1889-95.

63. Nulman I, Koren G, Rovet J, Barrera M, Pulver A, Streiner D, et al. Neurodevelopment of children following prenatal exposure to venlafaxine, selective serotonin reuptake inhibitors, or untreated maternal depression. Am J Psychiatry 2012; 169:1165-74.

64. Casper RC, Fleisher BE, Lee-Ancajas JC, Gilles A, Gaylor E, DeBattista A, et al. Follow-up of children of depressed mothers exposed or not exposed to antidepressant drugs during pregnancy. J Pediatr 2003; 142:402-8.

65. Austin MP, Karatas JC, Mishra P, Christl B, Kennedy D, Oei J. Infant neurodevelopment following in utero exposure to antidepressant medication. Acta Paediatr 2013; 102:1054-9.

66. Johnson KC, Smith AK, Stowe ZN, Newport DJ, Brennan PA. Preschool outcomes following prenatal serotonin reuptake inhibitor exposure: differences in language and behavior, but not cognitive function. J Clin Psychiatry 2016; 77:e176-82.

67. Hermansen TK, Røysamb E, Augusti EM, Melinder A. Behavior and inhibitory control in children with prenatal exposure to antidepressants and medically untreated depression. Psychopharmacology (Berl) 2016; 233:1523-35.

68. El Marroun H, White TJ, Fernandez G, Jaddoe VW, Verhulst FC, Stricker BH, et al. Prenatal exposure to selective serotonin reuptake inhibitors and non-verbal cognitive functioning in childhood. J Psychopharmacol 2017; 31:346-55

69. Viktorin A, Uher R, Kolevzon A, Reichenberg A, Levine SZ, Sandin S. Association of antidepressant medication use during pregnancy with intellectual disability in offspring. JAMA Psychiatry 2017; 74:1031-8.

70. Handal M, Skurtveit S, Roth C, HernandezDiaz S, Selmer R. Prenatal exposure to folic acid and antidepressants and language development: a population-based cohort study. J Clin Psychopharmacol 2016; 36:333-9.
71. Reebye PN, Morison SJ, Panikkar H, Misri S, Grunau RE. Affect expression in prenatally psychotropic exposed and nonexposed mother-infant dyads. Infant Ment Health J 2002; 23:403-16.

72. Casper RC, Gilles AA, Fleisher BE, Baran J, Enns G, Lazzeroni LC. Length of prenatal exposure to selective serotonin reuptake inhibitor (SSRI) antidepressants: effects on neonatal adaptation and psychomotor development. Psychopharmacology (Berl) 2011; 217:211-9.

73. Batton B, Batton E, Weigler K, Aylward G, Batton $\mathrm{D}$. In utero antidepressant exposure and neurodevelopment in preterm infants. Am J Perinatol 2013; 30:297-301.

74. Santucci AK, Singer LT, Wisniewski SR, Luther JF, Eng HF, Dills JL, et al. Impact of prenatal exposure to serotonin reuptake inhibitors or maternal major depressive disorder on infant developmental outcomes. J Clin Psychiatry 2014; 75:1088-95.

75. Skurtveit S, Selmer R, Roth C, HernandezDiaz S, Handal M. Prenatal exposure to antidepressants and language competence at age three: results from a large population-based pregnancy cohort in Norway. BJOG 2014; 121:1621-31.

76. Pedersen LH, Henriksen TB, Bech BH, Licht RW, Kjaer D, Olsen J. Prenatal antidepressant exposure and behavioral problems in early childhood: a cohort study. Acta Psychiatr Scand 2013; 127:126-35.

77. Grzeskowiak LE, Morrison JL, Henriksen TB, Bech BH, Obel C, Olsen J, et al. Prenatal antidepressant exposure and child behavioural outcomes at 7 years of age: a study within the Danish National Birth Cohort. BJOG 2016; 123:1919-28.

78. Hanley GE, Brain U, Oberlander TF. Prenatal exposure to serotonin reuptake inhibitor antidepressants and childhood behavior. Pediatr Res 2015; 78:174-80.

79. Oberlander TF, Reebye P, Misri S, Papsdorf M, Kim J, Grunau RE. Externalizing and attentional behaviors in children of depressed mothers treated with a selective serotonin reuptake inhibitor antidepressant during pregnancy. Arch Pediatr Adolesc Med 2007; 161:22-9.

80. Misri S, Reebye P, Kendrick K, Carter D, Ryan $\mathrm{D}$, Grunau RE, et al. Internalizing behaviors in 4-year-old children exposed in utero to psychotropic medications. Am J Psychiatry 2006; 163:1026-32.

81. Lupattelli A, Wood M, Ystrom E, Skurtveit $\mathrm{S}$, Handal M, Nordeng H. Effect of time-dependent selective serotonin reuptake inhibitor antidepressants during pregnancy on behavioral, emotional, and social development in preschool-aged children. J Am Acad Child Adolesc Psychiatry 2018; 57:200-8.

82. Grote NK, Bridge JA, Gavin AR, Melville JL, Iyengar S, Katon WJ. A meta-analysis of depression during pregnancy and the risk of preterm birth, low birth weight, and intrauterine growth restriction. Arch Gen Psychiatry 2010; 67:1012-24. 
83. Staneva A, Bogossian F, Pritchard M, Wittkowski A. The effects of maternal depression, anxiety, and perceived stress during pregnancy on preterm birth: a systematic review. Women Birth 2015; 28:17993.

84. Gentile S. Untreated depression during pregnancy: short- and long-term effects in offspring. A systematic review. Neuroscience 2017; 342:154-66.

85. Flint J, Kendler KS. The genetics of major depression. Neuron 2014; 81:1214.

86. Wray NR, Ripke S, Mattheisen M, Trzaskowski M, Byrne EM, Abdellaoui A, et al. Genomewide association analyses identify 44 risk variants and refine the genetic architecture of major depression. Nat Genet 2018; 50:668-81.

87. Kingston D, Tough S, Whitfield H. Prenatal and postpartum maternal psychological distress and infant development: a systematic review. Child Psychiatry Hum Dev 2012; 43:683-714.

88. Sansone RA, Sansone LA. Antidepressant adherence: are patients taking their medications? Innov Clin Neurosci 2012; 9:41-6.

89. Chapman SC, Horne R. Medication nonadherence and psychiatry. Curr Opin Psychiatry 2013; 26:446-52.

90. Matsui D. Adherence with drug therapy in pregnancy. Obstet Gynecol Int 2012; 2012:796590.

91. Gentile S, Galbally M. Prenatal exposure to antidepressant medications and neurodevelopmental outcomes: a systematic review. J Affect Disord 2011; 128:1-9.

92. Suri R, Hellemann G, Stowe ZN, Cohen LS, Aquino A, Altshuler LL. A prospective, naturalistic, blinded study of early neurobehavioral outcomes for infants following prenatal antidepressant exposure. J Clin Psychiatry 2011; 72:1002-7.

93. Anderson PJ, Burnett A. Assessing developmental delay in early childhood - concerns with the Bayley-III scales. Clin Neuropsychol 2017; 31:371-81.

94. Johnson S, Moore T, Marlow N. Using the Bayley-III to assess neurodevelopmental delay: which cut-off should be used? Pediatr Res 2014; 75:670-4.

95. Harris SR, Langkamp DL. Predictive value of the Bayley mental scale in the early detection of cognitive delays in high-risk infants. J Perinatol 1994; 14:275-9.

96. Heinonen E, Szymanska-von Schultz B, Kaldo V, Nasiell J, Andersson E, Bergmark M, et al. MAGDALENA: study protocol of a randomised, placebo-controlled trial on cognitive development at 2 years of age in children exposed to SSRI in utero. BMJ Open 2018; 8:e023281.
97. Singal D, Chateau D, Dahl M, Katz L, Ruth C, Wall-Wieler E, et al. In utero SSRI and SNRI exposure and the risk of neurodevelopmental outcomes in children: a population-based retrospective cohort study utilizing linked administrative data. Pharmacoepidemiol Drug Saf 2018; 27 Suppl 2:240-1.

98. Salisbury AL, Wisner KL, Pearlstein T, Battle CL, Stroud L, Lester BM. Newborn neurobehavioral patterns are differentially related to prenatal maternal major depressive disorder and serotonin reuptake inhibitor treatment. Depress Anxiety 2011; 28:1008-19.

99. Weikum WM, Oberlander TF, Hensch TK, Werker JF. Prenatal exposure to antidepressants and depressed maternal mood alter trajectory of infant speech perception. Proc Natl Acad Sci U S A 2012; 109 Suppl 2:17221-7.

100. Hanley GE, Brain U, Oberlander TF. Infant developmental outcomes following prenatal exposure to antidepressants, and maternal depressed mood and positive affect. Early Hum Dev 2013; 89:519-24.

101. de Vries NK, van der Veere CN, Reijneveld SA, Bos AF. Early neurological outcome of young infants exposed to selective serotonin reuptake inhibitors during pregnancy: results from the observational SMOK study. PLoS One 2013; 8:e64654.

102. Eriksen HL, Kesmodel US, Pedersen LH, Mortensen EL. No association between prenatal exposure to psychotropics and intelligence at age five. Acta Obstet Gynecol Scand 2015; 94:501-7.

103. Brown AS, Gyllenberg D, Malm H, McKeague IW, Hinkka-Yli-Salomäki S, Artama M, et al. Association of selective serotonin reuptake inhibitor exposure during pregnancy with speech, scholastic, and motor disorders in offspring. JAMA Psychiatry 2016; 73:1163-70.

104. Handal M, Skurtveit S, Furu K, HernandezDiaz S, Skovlund E, Nystad W, et al. Motor development in children prenatally exposed to selective serotonin reuptake inhibitors: a large population-based pregnancy cohort study. BJOG 2016; 123:1908-17.

105. Salisbury AL, O'Grady KE, Battle CL, Wisner KL, Anderson GM, Stroud LR, et al. The roles of maternal depression, serotonin reuptake inhibitor treatment, and concomitant benzodiazepine use on infant neurobehavioral functioning over the first postnatal month. Am J Psychiatry 2016; 173:147-57.

106. Hermansen TK, Yrttiaho S, Røysamb E, Melinder A. Perceptual interference processing in preschool children, with and without prenatal exposure to selective serotonin reuptake inhibitors. Psychopharmacology (Berl) 2017; 234:339-51. 


\section{Resumo}

O estudo teve como objetivo investigar se a exposição intrauterina a antidepressivos (ADs) aumenta o risco de transtornos do espectro autista (TEA), transtorno de déficit de atenção e hiperatividade (TDAH), esquizofrenia e outros transtornos mentais e déficits cognitivos e de desenvolvimento em lactentes e pré-escolares. Foram realizadas buscas nas bases PubMed, EMBASE e BIREME/ $B V S$ para identificar estudos sobre associações entre o uso de ADs durante a gestação e transtornos de neurodesenvolvimento e psiquiátricos. Vinte estudos trataram de riscos de TEA e/ou TDAH, enquanto 30 focaram em déficits cognitivos e de desenvolvimento em lactentes ou pré-escolares. A maioria dos estudos não detectou associação entre AD na gestação e TEA, depois de ajustar as razões de risco para depressão ou outros transtornos psiquiátricos maternos. Alguns estudos mostraram que a depressão materna, quer tratada ou não, aumenta o risco de TEA. Sete entre oito estudos não detectaram aumento de risco de TDAH associado à exposição intrauterina a inibidores seletivos da recaptação da serotonina, o AD mais comumente utilizado. Não foram encontradas evidências consistentes entre o uso de AD na gestação e déficits de desenvolvimento neurocognitivo em lactentes ou pré-escolares. Em quase todos os estudos, permaneceu um confundimento residual por indicação (gravidade da depressão). A revisão sistemática não encontrou evidências consistentes de que os ADs na gestação aumentassem o risco de TEA, TDAH ou déficits de desenvolvimento neurocognitivo. Entretanto, alguns estudos evidenciaram que a depressão materna aumenta o risco de TEA.

Antidepressivos; Depressão; Transtorno do Espectro Autista; Transtorno do Déficit de Atenção com Hiperatividade; Gravidez

\section{Resumen}

Este estudio investigó si la exposición prenatal a antidepresivos (ADs) incrementa los riesgos de trastornos del espectro autista (TEA), trastornos de déficit de atención/hiperactividad (TDAH), esquizofrenia, así como otras enfermedades mentales, cognitivas, $y$ déficits en el desarrollo de niños de primaria o preescolares. Se consultaron las bases de datos PubMed, EMBASE, BIREME/ $B V S$ para identificar estudios de asociaciones de ADs durante el embarazo con trastornos de desarrollo neurológico y psiquiátricos. Veinte estudios estaban centrados en riesgos de TEA y/o TDAH, mientras que 30 se centraron en déficits de desarrollo y cognitivos en niños de primaria o preescolares. La mayor parte de los estudios no detectaron asociación de AD, durante la etapa prenatal, con TDA tras el ajuste de las ratios de riesgo para depresión materna o trastornos psiquiátricos. Algunos estudios mostraron que la depresión materna, independientemente de si es tratada o no, incrementó los riesgos de TEA. Siete de los 8 estudios no encontraron un incremento en el riesgo de TDAH, asociado con la exposición prenatal a inhibidores selectivos de la recaptación de serotonina, el antidepresivo más usado habitualmente durante el período prenatal. No se encontraron evidencias consistentes relacionando $A D$ durante el embarazo y déficits en el desarrollo neurocognitivo de niños de primaria o preescolares. En casi todos los estudios hubo una desviación residual señalada como gravedad de la depresión. Esta revisión sistemática no halló evidencias consistentes, sugiriendo que el consumo de ADs durante el embarazo incremente el riesgo de TEA, TDAH, y déficits en el desarrollo neurocognitivo. Algunos estudios, no obstante, encontraron evidencias de que la depresión materna incrementa riesgos de TEA.

Antidepresivos; Depresión; Trastorno del Espectro Autista; Trastorno por Déficit de Atención con Hiperactividad; Embarazo
Submitted on $18 / \mathrm{Feb} / 2019$

Final version resubmitted on 12/Nov/2019

Approved on 02/Dec/2019 